\title{
Modeling fracturing pressure parameters in predicting injector performance and permeability damage in subsea well completion multi-reservoir system
}

\author{
Kingsley E. Abhulimen ${ }^{1}$ S. Fashanu ${ }^{2}$ Peter Idialu ${ }^{2}$
}

Received: 22 March 2017/ Accepted: 1 July 2017/Published online: 1 August 2017

(c) The Author(s) 2017. This article is an open access publication

\begin{abstract}
The significance of fracturing parameters which are aquifer integrity, rock properties, thermal stress, fracturing pressure and produced water quality to alter permeability damage, cake formation and injectivity performance was highlighted in a robust improved internal filtrationhydraulic model and permeability reduction model incorporating a $R_{\mathrm{AT}}(c)$ function. The studied system is an injection well multi-reservoir formations. Field data obtained from the log and field reports and improved model were used to simulate injector, fracturing and permeability damage performance. Thus, data requirements in the $R_{\mathrm{AT}}(c)$ function which are rock properties, water quality, aquifer integrity, fractures rates and pressures parameters were assessed for its impact on injector performance and permeability damage simulated in MATHLAB and COMSOL multi physics environment. The profile of injector performance and damage reservoir permeability to changes in rock properties and aquifer integrity were demonstrated to have a profound influence on both fracturing phenomena. Thus, sustainable re-injection scheme was shown as a direct consequence of rock mechanics parameters, well hydraulics aquifer integrity that largely depends on the initial concentration of active constituents of the produced water as well as physic-chemical properties of the host aquifer.
\end{abstract}

Keywords Re-injection · Fracturing · Rock properties · Permeability damage $\cdot$ Acquifer

Kingsley E. Abhulimen

syntechsysworld@gmail.com

1 Department of Chemical and Petroleum Engineering, University of Lagos, Lagos, Nigeria

2 Department of Systems Engineering, University of Lagos, Lagos, Nigeria

\author{
List of symbols \\ $\mathrm{S}_{\mathrm{T}} \quad$ Skin factor \\ $\mu \quad$ Viscosity \\ $P_{\text {inj }} \quad$ Injection Pressure \\ q Flow rate $\left(\mathrm{m}^{3} / \mathrm{s}\right)$ \\ $\mathrm{k} \quad$ Permeability \\ $\mathrm{k}_{\sigma} \quad$ Permeability damage factor \\ $\eta \quad$ Total collision probability \\ $\eta 1$ Collision probability due to interception \\ $\eta \mathrm{D} \quad$ Collision probability due to diffusion \\ $\eta \mathrm{lm} \quad$ Collision Probability due to impaction \\ $\eta \mathrm{s} \quad$ Collision probability due to sedimentation \\ $\eta \mathrm{E} \quad$ Collision probability due to surface forces \\ dp Particle diameter \\ dg Grain diameter \\ $\phi \quad$ Effective porosity \\ $\rho_{p} \quad$ Particle density \\ $\rho_{f} \quad$ Fluid density \\ $U, u \quad$ Darcy's velocity \\ g Gravity acceleration $\left(\mathrm{m} / \mathrm{s}^{2}\right)$ \\ $\mathrm{T} \quad$ Absolute temperature $\left(\mathrm{K},{ }^{\circ} \mathrm{C}\right)$ \\ $C(r, t) \quad$ Volumetric concentrations of suspended particles \\ (ppm) \\ $\sigma(r, t)$ Volumetric concentrations of the deposited \\ particles (ppm) \\ ko Absolute permeability \\ $\lambda \quad$ Filtration coefficient \\ L Depth of the porous media \\ $\varepsilon_{r} \quad$ Scaled length in radial direction \\ $\varepsilon_{z} \quad$ Scaled length in axial direction \\ $t \quad$ Time (yrs) \\ $\tau \quad$ Scaled time \\ $\in \quad$ Scaled concentration of suspended solids \\ S Scaled concentration of deposited particles
}


$\lambda_{o} \quad$ Initial filtration coefficient

$\alpha_{c} \quad$ Clean bed collision efficiency

I Injectivity index

J Inverse of Injectivity Index

$\mathrm{Tr} \quad$ Transition time

$\mathrm{n} \quad$ Number of particles attached to one grain

$J_{d} \quad$ Impedance during one phase suspension flow

$K_{\text {ror }} \quad$ Relative permeability of residual oil

$m \quad$ Slope of Impedance straight line during deep bed filtration for one Phase suspension flow

$m c \quad$ Slope of Impedance straight line during external cake formation for one phase suspension flow

$\mathrm{p} \quad$ Pressure (M/LT2, Pa)

$q \quad$ Total flow rate per unit reservoir thickness, $\mathrm{L}^{2} / \mathrm{T}$

$r \quad$ Reservoir radius $(\mathrm{L}, \mathrm{m})$

$r_{\mathrm{w}} \quad$ Well radius $(\mathrm{L} / \mathrm{m})$

$r d \quad$ Damage zone radius $(\mathrm{L}, \mathrm{m})$

Rc Contour radius $(\mathrm{L}, \mathrm{m})$

Sor Residual oil saturation

Swi Initial water saturation

$T \quad$ Time (T, s)

$T \quad$ Dimensionless time

$T_{\text {tr }} \quad$ Dimensionless transition time

$U$ Total flow velocity ( $\mathrm{L} / \mathrm{T}, \mathrm{m} / \mathrm{s}$ )

$\alpha \quad$ Critical porosity fraction

$\beta \quad$ Formation damage coefficient

$\phi \quad$ Porosity

\section{Definition of terms and acronyms}

Produced water

Produced water re-injection

Non-fresh water

hydrocarbon aquifer

Reservoir

Formation

Produced water constituents

Injection pipe

Well bore
Water associated with crude oil exploration and production

Sending back produced water from the surface into the subsurface

Crude oil bearing formation

A permeable subsurface rock that contains petroleum

Refers to the reservoir bearing fluids e.g. oil, gas and water

Heavy metals, suspended solids, dissolved solids, hydrocarbon traces etc.

Produced water transfer medium from surface to subsurface

Point of contact of injection pipe with formation/ reservoir
Deep bed filtration

Injectivity decline

Formation damage

Adsorption kinetics

Hydrodynamic

dispersion

Geochemical reaction

Colloids

Scales

Cakes

Geomechanics

Corrosion

Souring

Representative elementary volume

Isotherms

Finite element method
The flow and deposition of particles in the rock matrix Index signifying the change in the injection rate of the injected fluid

Reduction in aquifer properties that are solely responsible for the transmissibility of reservoir fluids through the pore spaces (fracture in internal walls of the aquifer)

Attraction and retention of particle to the surface grain and the preference of this particle for a particular site within the reservoir

Is a term used to include both diffusion and dispersion of particles within a medium

This is the interaction of species constituents in the produced water and the formation of the host aquifer

Colloidal particles are suspended particles carried in the fluid stream

Result of nucleation of colloids

Deposition of scales in pore sites is referred to as cakes Involves the geologic study of the behavior of soil and rock

Loss in metal due to degradation, erosion or prevailing ambient conditions

Acidic smell/taste characteristic

A pictured or drawn shape representative of the actual shape. Used in solving mathematical problems

Equations considered at constant temperature

Numerical method of solution whereby a problem is characterized 
by boundaries and solved within these boundaries

PW

PWRI

EOR

$\mathrm{E} \& \mathrm{P}$

REV

TVD

BHP

\section{Introduction}

Produced water re-injection in multi-reservoir and hydrocarbon aquifer systems above fracturing pressure is a necessary water flood strategy commonly employed for disposal of produced water in subsea well peripheral water flood project. There are several leading publications in the field produced water injection modeling, fractured modeling, injectivity decline and their outcome of particulate mechanics and flow studies (Pang and Sharma 1997; Barkman and Davidson 1972; Wennberg and Sharma 1997).

Regardless of the source, produced water handling and injection is still the single biggest operating costs for producers in mature fields (Ajay and Sharman 2007; Salehi and Settari 2008). Studies related to investigation of rate of fracture height and length growth due to injection above fracture pressure are required to evaluate injection strategies where necessary (Prasad et al. 1999). Water injection is the outcome of stricter offshore regulatory requirements accounting for 500 million bbl. of water/day injected into the subsurface formation, annual operating costs in the range of $\$ 100$ billion US Dollars.

Current models for predicting internal filtration and injectivity decline in water injection studies for secondary recovery were only limited to mass balance of suspended solids, settling particles equation, particle capture kinetics and Darcy's law accounting for permeability damage to particle retention. Other analytical models are limited to both particle retention and water-oil mobility alteration (Belfort et al. 1994; McDowell-Boyer et al. 1986).

Improved models and field data to describe the role of geochemical reaction, adsorption-scale kinetics were recently published to impact cake formation, permeability damage and injection performance (Obe et al. 2017). Nonetheless, the wellestablished field data for fractured modeling in most cases show more than these parameters including filtration coefficient $\lambda$ characterizing the intensity of the particle capture by the porous rock, while formation damage coefficient $\beta$ shows permeability decrease due to particle capture (Pang and Sharman 1994; AlAbduwani et al. 2001; Guo 2000; Meyer et al. 2003a, b).
Formation damage has been studied under two subject domains; internal filtration and external cake build up. Several articles have provided models and understanding in the field of injectivity decline for characterization of the formation damage system and consequent well behavior prediction. The combined effect of particle suspension injection and total oil-water mobility variation on well injectivity was studied (Altoef et al. 2004). Explicit formulas for injectivity decline due to both effects were derived and applied their model for a deep water offshore reservoir.

The filtration and formation damage coefficients and filter cake permeability from the well injectivity history were determined from the linear dependence of impedance index (the inverse of injectivity index) on injected water volume for deep bed filtration and external cake formation. Researchers considered the effect of particle/pore size distribution, injected solid concentration, wellbore narrowing, particle invasion (Pang and Sharma 1997; Barkaman and Davidson 1972; Donaldson et al. 1977), but fell short to highlight its impact on injector performance and permeability damage and fail to relate rock in situ stresses, aquifer integrity and produced water quality as important in these assessments, which is the objective of our study.

Several other models exist to describe fracturing, injectivity decline, formation damage, particulate mechanics and this have been published elsewhere (AbouSayed et al. 2005, 2007; Al-Abduwani 2005; Bedrikovetsky et al. 2007; Chang 1985; Clifford et al. 1991; Davidson 1979; De Zwart 2007; Dong et al. 2010; Donaldson et al. 1977; Doresa et al. 2012; Farajzadeh 2002; Faruk 2010; Folarin et al. 2013; Furtado et al. 2005; Gong et al. 2013; Greenhill 2002; Guedes et al. 2006; Hustedt et al. 2006; Iwasaki 1937; Khatib 2007; Khodaverdian et al. 2009; Lawal and Vesovic 2010; Lawal et al. 2011; Li et al. 2011, 2012; Ojukwu and van den Hoek 2004; Sahni and Kovacevich 2007; Shuler and Subcaskey 1997; Souza et al. 2005; Todd 1979; Van den Hoek et al. 1996; Wang and Le 2008; Wang et al. 2011; Sharma et al. 2000; Yerramilli et al. 2013; Zeinijahromi et al. 2011; Zhang et al. 1993).

In this study, the significance of rock stresses mechanics, aquifer integrity and produced water quality in altering permeability damage, fracturing, cake formation and injectivity decline were highlighted in a robust improved internal filtration-hydraulic model. Thus, our solution accounted for suspended particle propagation $C(X, T)$, retained particle accumulation $S(X, T)$, aquifer integrity related to grain/particle size ration and "In Situ Rock Stress and Wellbore Stability highlighted in the Frade Field, Brazil," Frade CPDEP Phase 2 report DR-AP-RP-021,209 by GeoMechanics International, Inc. (Guo 2000, Meyer et al. 2003a, b). 


\section{Reduced model for PWRI and fracturing performance}

As an improvement over the filtration model for cake formation, fracturing well hydraulics and aquifer integrity residual oil mobility and correction for good completion geometry, rock mechanics formation damage coefficient including geochemical reaction, leak off parameters and retention kinetics were introduced as $R_{A T}$.

$$
\begin{aligned}
& \frac{\partial \phi C}{\partial t}+\left(U_{r} \frac{\partial C}{\partial r}+\frac{U_{r} C}{r}+U_{z} \frac{\partial C}{\partial z}\right) \\
& -\left(\left(D_{r} \frac{\partial^{2} C}{\partial r^{2}}\right)+\frac{1}{r} D_{r} \frac{\partial C}{\partial r}+D_{z} \frac{\partial^{2} C}{\partial z^{2}}\right) \\
& =\frac{\partial \sigma}{\partial t}+R_{A T}
\end{aligned}
$$

$R_{A T}(c, t, \phi)=\left(\sum_{i=0}^{N} w_{i} R_{F e f f_{i}}\right)=\left(1-\sum^{N} k_{o r i}\right) \frac{\partial \phi C}{\partial t}$, a variable that is a function of concentration, transition time to cake formation, and effective porosity that highlights the contribution of rock properties, aquifer integrity, fracturing pressure and water quality related to impacts in geochemical reaction and adsorption kinetics.

Subject to the Robin type boundary condition

$C(r=0, t)=C_{o}$

$C\left(r=r_{n}, z=z_{n}, t_{n}=0\right)=0^{\prime}$

$\left[\frac{\partial C}{\partial r}\right]_{r=R c, t}=0\left[\frac{\partial C}{\partial z}\right]_{r=Z c, t}=0$

Dimensionless form of the boundary condition

$$
\begin{aligned}
& \zeta\left(\varepsilon_{r}=0, \tau \geq 0\right)=1 \\
& \zeta\left(\varepsilon_{r}=1, \varepsilon_{z}=1, t_{n}=0\right)=0 \\
& {\left[\left(\frac{c_{o}}{R_{o}}\right) \frac{\partial \zeta}{\partial \varepsilon_{\bar{r}}}\right]_{\varepsilon_{r}=1, \tau}=0\left[\left(\frac{c_{o}}{L}\right)\left(\frac{\partial \zeta}{\partial \varepsilon_{\bar{z}}}\right)\right]_{z=1, \tau}=0}
\end{aligned}
$$

The generalized equations of the internal filtration model are converted to dimensionless form parameters define as follows:

$$
\begin{aligned}
& \varepsilon_{\bar{r}}=\frac{r}{R_{c}} \\
& \varepsilon_{\bar{z}}=\frac{z}{L} \\
& \tau=\left(\frac{v}{\phi l}\right) t \\
& S=\frac{\sigma}{\phi c_{o}} \\
& \zeta=\frac{c}{c_{o}} \\
& \Lambda(S)=\lambda(\sigma) L
\end{aligned}
$$

$r_{D}=\frac{q_{d i}}{\phi c_{d i}}$

Now $: \frac{\partial c}{\partial t}=\frac{\partial c}{\partial \tau} \frac{\partial \tau}{\partial t}=\left(\frac{v}{\phi L}\right) c_{o} \frac{\partial \zeta}{\partial \tau}$

$\left(\frac{v}{\phi L}\right) c_{o} \frac{\partial \zeta}{\partial \tau}=\frac{\partial \zeta}{\partial \tau^{*}}$

where: $\frac{\partial \tau}{\partial t}=\left(\frac{v}{\phi L}\right)$

Equation 1 is re-expressed in dimensionless form as:

$$
\begin{aligned}
& \left(\frac{\partial \zeta}{\partial \tau^{*}}-\left(\frac{\partial S}{\partial \tau^{*}}+\frac{\partial \Psi_{D}}{\partial \tau^{*}}\right)+\frac{\partial \Psi_{r}}{\partial \tau^{*}}+\frac{\partial \Psi_{k f f}}{\partial \tau}\right)+\alpha_{1}(v)\left(\frac{\partial \zeta}{\partial \varepsilon_{r}}\right) \\
& \quad+\alpha_{2}(v) \frac{\zeta}{\varepsilon_{r}}+\alpha_{3}(v) \frac{\partial \zeta}{\partial \varepsilon_{z}} \\
& \quad=\alpha_{4}(v) \frac{\partial^{2} \zeta}{\partial r^{2}}+\alpha_{5}(v)\left(\frac{\partial \zeta}{\partial \varepsilon_{r}}\right)+\alpha_{6}(v)\left(\frac{\partial^{2} \zeta}{\partial \varepsilon_{\bar{z}}^{2}}\right)
\end{aligned}
$$

The partial differential equations are solved by the Tridiagonal Matrix Algorithm (TDMA) method. In the model, a second-order six-point implicit finite scheme has been used to obtain a numerical of the governing equations involving the concentration field:

$$
\begin{aligned}
& \alpha_{1}^{\prime} \zeta_{i j k+1}+\alpha_{2}^{\prime} \zeta_{i+1 j k+1}+\alpha_{3}^{\prime} \zeta_{i j+1 k+1}+\alpha_{4}^{\prime} \zeta_{i-1 j k+1}+\alpha_{5}^{\prime} \zeta_{i j-1, k+1} \\
& \quad=\alpha_{6}^{\prime} \zeta_{i j k}+\hat{a}_{r}\left(\Psi_{r i j k+1}-\Psi_{r i j k}\right)-\hat{a}_{d}\left(\Psi_{d i j k+1}-\Psi_{d i j k}\right)
\end{aligned}
$$

where:

$$
\begin{aligned}
& \alpha_{1}^{\prime}=1-\Delta \tau^{*}\left(\frac{\alpha_{1}}{\Delta \epsilon_{\bar{r}}}+\frac{\alpha_{3}}{\Delta \epsilon_{\bar{z}}}-\frac{2 \alpha_{4}}{\left(\Delta \epsilon_{\bar{r}}\right)^{2}}-\frac{2 \alpha_{6}}{\left(\Delta \epsilon_{\bar{z}}\right)^{2}}\right) \\
& \alpha_{2}^{\prime}=\left(\frac{\alpha_{1} \Delta \tau^{*}}{\Delta \epsilon_{\bar{r}}}-\frac{\alpha_{4} \Delta \tau^{*}}{\left(\Delta \epsilon_{r}\right)^{2}}+\frac{\alpha_{5} \Delta \tau^{*}}{\Delta \epsilon_{r}}\right)
\end{aligned}
$$

$\alpha_{3}^{\prime}=\left(\frac{\alpha_{3} \Delta \tau^{*}}{\Delta \epsilon_{z}}-\frac{\alpha_{6} \Delta \tau^{*}}{\left(\Delta \epsilon_{z}\right)^{2}}\right)$

$\alpha_{4}^{\prime}=\left(\frac{\alpha_{4} \Delta \tau^{*}}{\left(\Delta \epsilon_{r}\right)^{2}}\right)$

$\alpha_{5}^{\prime}=\left(\frac{\alpha_{6} \Delta \tau^{*}}{\left(\Delta \epsilon_{z}\right)^{2}}\right)$

$\alpha_{6}^{\prime}=1-\Delta \tau^{*}\left(\alpha_{o}+\alpha_{2}\right)$

where:

$\alpha_{1}(v)=\left(\frac{v_{r}}{v}\right)\left(\frac{L}{R_{o}}\right)$

$\alpha_{2}(v)=\left(\frac{v_{r}}{v}\right)\left(\frac{L}{R_{o}}\right)\left(\frac{1}{\varepsilon_{r}}\right)$ 
$\alpha_{3}(v)=\frac{v_{z}}{v}$

$\alpha_{4}(v)=\left(\frac{D_{e r}}{v}\right)\left(\frac{L}{R_{o}^{2}}\right)$

$\alpha_{5}(v)=\left(\frac{D_{e r}}{v}\right)\left(\frac{L}{R_{o}^{2}}\right)\left(\frac{1}{\varepsilon_{r}}\right)$

$\alpha_{6}(v)=\left(\frac{D_{e z}}{v}\right)\left(\frac{1}{L}\right)$

$\tau^{*}=\left(\frac{1}{\left(1-k_{o r} R_{A T}\right)}\right)$

For the implicit finite difference scheme, multiply by $\Delta \tau^{*}$ and rearranging yields;

$$
\begin{aligned}
& \alpha_{1}^{\prime} \zeta_{i j k+1}+\alpha_{2}^{\prime} \zeta_{i+1 j k+1}+\alpha_{3}^{\prime} \zeta_{i j+1 k+1}+\alpha_{4}^{\prime} \zeta_{i-1 j k+1}+\alpha_{5}^{\prime} \zeta_{i j-1, k+1} \\
& \quad-\alpha_{6}^{\prime} \zeta_{i j k} \\
& \quad=\hat{a}_{r}\left(\Psi_{r i j k+1}-\Psi_{r i j k}\right)-\hat{a}_{d}\left(\Psi_{d i j k+1}-\Psi_{d i j k}\right)
\end{aligned}
$$

Rearranging, for $i=1, n, k=1, n$, for $j=1, n$, then the defining matrix equation

$$
A \Omega_{i k+1}+B \Omega_{i-1 k+1}+C \Omega_{i+1 k+1}=D \Omega_{i k}+\Delta \bar{\Xi}_{i k}+\bar{c}_{o}+\bar{d}_{0}
$$

Prediction of geomechanical rock failure derived from rock stress factors evolves from the Mohr-Coulomb failure criterion. Mechanical decementation responses are governed by a phenomenon called rock fracture arching which is the resistance to withhold forces applied due to mechanical and hydrodynamic stresses. Radial stress gradient is derived from one of the equations of equilibrium in spherical coordinates as presented in Eq. 35

$\frac{\partial \sigma_{r}}{\partial r}+\frac{1}{r}\left(2 \sigma_{r}-\sigma_{\theta}-\sigma_{\phi}\right)=0$

A simplified spherical symmetry of the stressed field was assumed such that two tangential stresses are equal that is: $\sigma_{\theta}=\sigma_{\phi}$ resulting Eq. 36

$\frac{\partial \sigma_{r}}{\partial r}+\frac{2}{r}\left(\sigma_{r}-\sigma_{\theta}\right)=0$

By Mohr-Coulomb criterion, radial and tangential stresses are related by:

$\sigma_{\theta}-P_{f}=C_{o}+\left(\sigma_{r}-P_{f}\right) \tan ^{2} \beta$

At the cavity wall, $P_{f}=P_{w}=\sigma_{r}$, therefore:

$\sigma_{\theta}-\sigma_{r}=C_{O}=2 S_{o} \tan \beta$

An expression for normal stress gradient is given by Eq. 3

$\left[\frac{\partial \sigma}{\partial r}\right]_{r=R_{c}}=\left[\frac{2 C_{o}}{r}\right]_{r=R_{c}}=\left[\frac{4 S_{o} \tan \beta}{r}\right]_{r=R_{c}}$ where $C_{\mathrm{o}}$ is uniaxial compressive strength. $S_{\mathrm{o}}$ is cohesive strength. $R_{\mathrm{c}}$ is cavity radius (Fig. 1).

The basic equations that for constituting rock fracture models are: (1) Mechanical equilibrium eq. (2) Constitutive equation for the porous medium. (3) Continuity equation for fluid. (4) Darcy's law. Extending the paradigm for rock fracture prediction models is progressed by a rock fracture production factor $k_{L}$ derived from the

Mohr-Coulomb Failure Criterion is segmented into three stages (1) Formation failure (2) Rock fracture erosion due to flow (3) Rock fracture transport (Fig. 2).

Rock failure occurs when the shear stress on a given plane within the rock reaches a critical value;

$\tau_{\max }=S_{o}+\sigma^{\prime} \tan \phi$

Figure 2 shows the angle $2 \beta$, which gives the position of the point where the Mohr's circle touches the failure line. Shear stress at this point of contact is given by Eq. 41:

$|\tau|=\frac{1}{2}\left(\sigma_{1}^{\prime}-\sigma_{3}^{\prime}\right)$

Normal stress is given by:

$\sigma^{\prime}=\frac{1}{2}\left(\sigma_{1}^{\prime}+\sigma_{3}^{\prime}\right)+\frac{1}{2}\left(\sigma_{1}^{\prime}-\sigma_{3}^{\prime}\right) \cos 2 \beta$

Also, $\beta$ and $\varphi$ are related thus:

$\beta=\frac{\pi}{4}+\frac{\phi}{2}$

$\beta$ is the angle of failure criterion. The maximum normal stress is related to the minimum normal stress

$\sigma_{1}^{\prime}=2 S_{o}\left(\frac{\cos \phi}{1-\sin \phi}\right)+\sigma_{3}\left(\frac{1+\sin \phi}{1-\sin \phi}\right)$

The maximum stress is further given by:

$\sigma_{1}^{\prime}=C_{o}+\sigma_{3}^{\prime} \tan ^{2} \beta$

Rock failure in petroleum production from mature fields represents significant equipment maintenance and work over costs challenges. Rock failure models documented in technical literature is solved using the mass balance equation of fluidized solids in conjunction with the

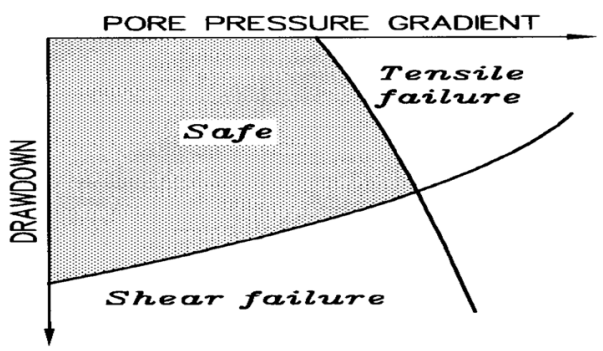

Fig. 1 Stability diagram for production cavities as reported by (Morita et al. 1987a, b: SPE) 
erosion criterion and mass balance of the flowing fluids. However, equilibrium equation and, therefore, the mechanical responses of the reservoir, are not well captured. Rock stress failure is a two-stage process. The first stage is fractured rock stone decementation. Before rock fracture stone is decemented, rock fracturing cannot occur. Simulation of aquifer decementation requires the solution of equilibrium equation along with a suitable constitutive equation. Models based on coupled erosion-geomechanical model concepts are limiting. Therefore, there must be two conditions to produce rock fractures: (1) rock failure is mainly determined by the rock shear stress, and (2) aquifer production flow rate is mainly controlled by the fluid shear stress. Equation 46 is the Mohr-coulomb criterion correlation use in determining the range of the failure plane for which rock fracture production can be predicted. Mohr-Coulomb model is extended using rock fracture factor, $K_{\mathrm{Ls}}$ in a defining equation, where rock fracturing factor of 0 represents (minimum threshold of failure or rock fracturing) and rock fracturing factor of 1 is maximum safe zone when $K_{\mathrm{L}}<0$ to limit extensive rock fracture data requirement in the development of predictive models:

Necessary condition for rock fracture is given by:

$k_{R s}=\left[1-\left(\frac{\tau_{\max }}{\text { Fluid Shear Stress } \tau_{P}+\text { Rock Shear Stress }|\tau|}\right)\right]$.

The rock shear stress $|\tau|$ and maximum shear stress $\tau_{\max }$ are represented by the Mohr-Coulomb Failure criterion

Sufficient condition for rock fracture is given when necessary condition is attained:

The fluid pressure shear stress $\tau_{p}$ derived from the Darcy equation greater that than rock stresses-maximum stresses lead to rock fracture occurring (Figs. 3, 4). Rock fracture is only produced when the fluid shear stress is greater than the residual stress from the maximum rock stress-rock shear stresses $0 \leq k_{\mathrm{fLs}} \leq 1$.

$$
\begin{aligned}
& k_{\mathrm{fs}}=\left[1-\left(\frac{\tau_{\mathrm{max}}-\text { Rock Shear Stress }|\tau|}{\text { Fluid Shear Stress } \tau_{P}}\right)\right] \\
& \tau_{p}=\hat{k}\left(k_{r} \nabla^{2} p+\nabla p \nabla k_{r}\right)
\end{aligned}
$$

The region of rock fracturing is represented as $\tau_{\max }-$ Rock Shear Stress $|\tau|<$ Fluid Shear Stress $\tau_{P}$,

$0 \leq k_{L s} \leq 1$

$-1 \leq k_{L s}<0$ is region of. $\tau_{\max }>$ (Rock Shear Stress $\left.|\tau|\right)$, $0 \leq k_{L s}<-m$ represents the region of no rock fracturing or safe region.

$$
\begin{aligned}
\tau_{\max }= & {[\text { Rock Shear Stress }|\tau|] } \\
& +\left[\text { Fluid Shear Stress } \tau_{P}\right]\left(1-k_{L s}\right) \\
S_{o}= & \tau_{\max }-\sigma^{\prime} \tan \phi
\end{aligned}
$$

where the fluid shear stress is computed from Eq. 14 becomes the sufficient condition

$\tau_{z}=\hat{k}\left(k_{r} \nabla^{2} p+\nabla p \nabla k_{r}\right)$

$|\tau|=\frac{1}{2}\left(\sigma_{1}^{\prime}-\sigma_{3}^{\prime}\right) \quad \sigma^{\prime}=\frac{1}{2}\left(\sigma_{1}^{\prime}+\sigma_{3}^{\prime}\right)+\frac{1}{2}\left(\sigma_{1}^{\prime}-\sigma_{3}^{\prime}\right) \cos 2 \beta$

In this paper, concept of rock failure factor or rock failure producing factor $\left(k_{\mathrm{LS}}\right)$ to predict and quantify rock fracture produced in a reservoir field leads to the conclusion that the rock fails when rock shear stress is greater than or equal to the maximum rock shear stress. This is a necessary condition for rock fracture production must be failure of the rock; i.e., the rock shear stress must be greater than or equal to the maximum shear stress. If this condition is not met, rock fracture cannot be produced, regardless of the value of fluid shear stress. Fluid shear stress mainly controls the rock fracture production rate and not the rock failure, and this becomes the sufficient condition that rock fracture is produced. Fluid shear stress can be considered at the sufficient condition for rock fracture flow; therefore:
Fig. 2 Mohr-coulomb criterion in $\tau-\sigma^{\prime}$ space, and Mohr's circle critical stress state

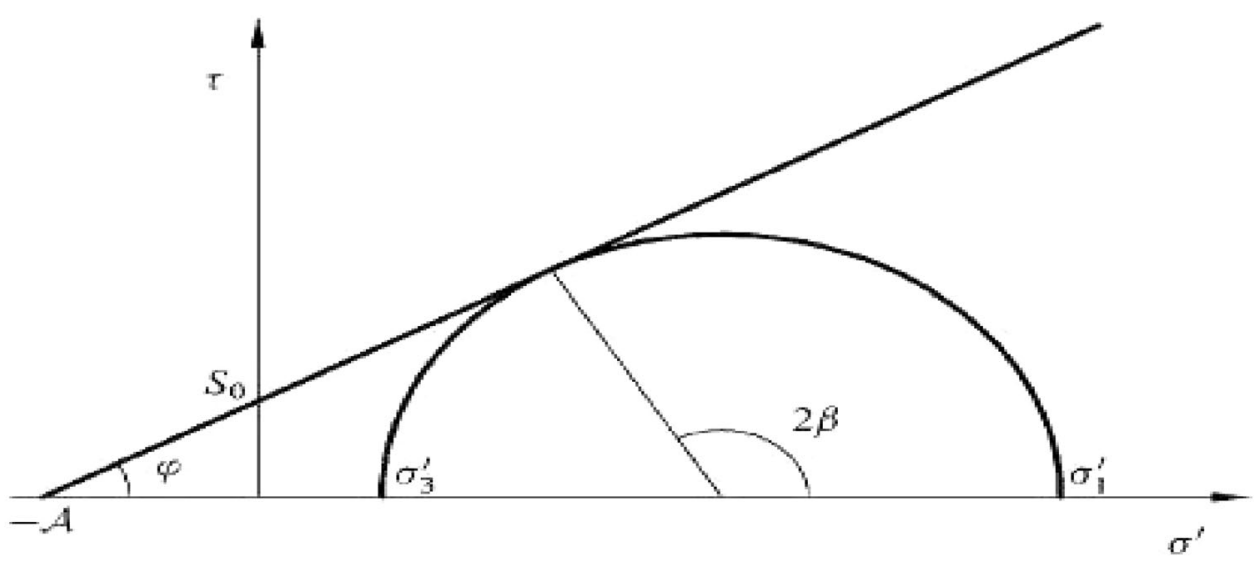




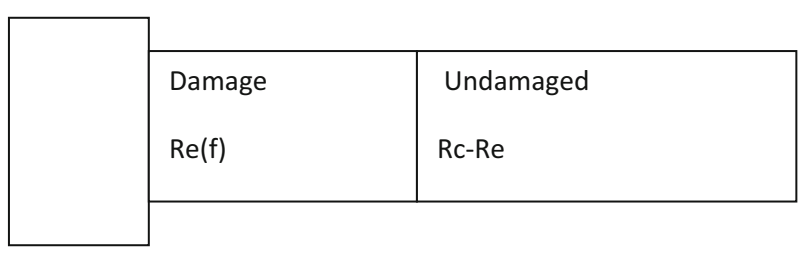

Fig. 3 Diagram for damage and undamage section of reservoir

1. The lowest fluid shear stress yields the most rock fracture propagation $\left(k_{\mathrm{LS}}=0\right.$, fluid shear stress $=0$ ) which leads to not much fluid flow.

2. The highest fluid shear stress yields the least rock fracture propagation $\left(k_{\mathrm{LS}}=1\right.$, fluid shear stress $\gg$ rock shear stress) which leads to more fluid flow

The most interesting result in the paper is that the value of fluid shear stress controls the rock fracture propagation rate. The combined effect of rock failure and fluid shear stress leads to rock failure propagation leading to fractured rocks.

\section{Permeability damage reduction model}

As particles are trapped in the pore throats permeability declines, which in return leads to a reduction in injectivity. Several relationships have been suggested to relate the decline in permeability to the concentration of deposited particles $(17,18)$. Wennberg and Sharma (1997) proposed a permeability reduction model starting with the Carman Kozeny equation:

$\kappa=\frac{\phi^{3}}{5(1-\phi)^{2}} \frac{1}{s^{2}} \frac{1}{\tau}$

Here, $S$ is the specific surface area based on the solids volume and $\tau$ is the tortuosity of the porous medium. They further postulate that the permeability reduction due to particle deposition can be split into 3 parts: reduced porosity, increased surface area and increased tortuosity. The reduced permeability model can thus be expressed as Eq. 54:

$\frac{k}{k_{0}}=k_{\mathrm{dp}} k_{\mathrm{ds}} k_{\mathrm{dt}}$

where

$k_{\mathrm{dp}}=\frac{\phi^{3}}{\phi_{0}^{3}} \frac{\left(1-\phi_{0}^{2}\right)}{\left(1-\phi^{2}\right)}$

$k_{d s}=\left(\frac{1+\sigma /\left(1-\varphi_{0}\right)}{1+\sigma /\left(1-\varphi_{0}\right)\left(d_{g} / d_{p}\right)}\right)^{2}$

$k_{d t}=\frac{1}{(1+\beta \sigma)}$

The damage factor $\beta$ accounts for trapped particles deposit in the pores. $B$ is normally greater than 0 .
The permeability distribution is determined by the extent and distribution of particles trapped in the pore spaces. Payatakes et al. indicate that the pressure drop increase is a linear function of the extent of the particle deposition in the case of dilute suspension injection. This suggests that the following equation holds for small particle sizes

$k(C)=\frac{k(x, t))}{k_{m}}=\frac{1}{1+\beta \sigma(x, t)}$

where $\beta$ is a constant and represents the damage factor.

The average dimensionless permeability between the injected face and the injection front of the core can be obtained by expanded model including the $R_{\mathrm{AT}}(c)$ function and permeability damage factor.

$k^{\prime}(C)=\frac{k(r, z, t)}{k_{m}}=\frac{K_{O} \cdot e^{-R_{\mathrm{AT}}}}{1+\beta \sigma}$

$K_{O}=k_{\mathrm{dp}} \cdot k_{\mathrm{ds}}$

where $\sigma$ can be determined by Eq. 42 below:

$\frac{\partial \sigma}{\partial t}=\lambda v C$

\section{Injectivity performance related to fracturing pressure}

The sustaining or fracturing pressure equation derived from mass balance injector-production performance is given as Eq. 43 below

$\rho c_{T} \frac{\partial \phi P}{\partial t}+\rho \nabla \lambda P=i-q_{i}$

$i=$ injection rate; $q_{i}=$ production rate

For cylindrical coordinates:

$\left(c_{T} \phi \frac{\partial P}{\partial t}+\lambda \nabla P\right)+\left(P c_{T}\left(\frac{\partial \phi}{\partial t}\right)+P \nabla \lambda\right)=\left(\frac{i-q_{i}}{\rho}\right)$

$\left(c_{T} \phi \frac{\partial P}{\partial t}+\lambda_{r} \frac{\partial P}{\partial r}+\lambda_{z} \frac{\partial P}{\partial z}+\frac{\lambda_{r} P_{r}}{r}\right)$

$+\left(P c_{T}\left(\frac{\partial \phi}{\partial t}\right)+P_{r} \frac{\partial \lambda}{\partial r}+P_{z} \frac{\partial \lambda}{\partial z}+\frac{\lambda_{r} P_{r}}{r}\right)$

$=\left(\frac{i-q_{i}}{\rho}\right)$

Measure of interconnectivity

$$
\begin{aligned}
& \frac{\partial \phi}{\partial t}=K_{I} \frac{\partial \lambda}{\partial t} \\
& \left(c_{T} \phi \frac{\partial P}{\partial t}+\lambda_{r} \frac{\partial P}{\partial r}+\lambda_{z} \frac{\partial P}{\partial z}+\frac{2 \lambda_{r} P_{r}}{r}\right) \\
& \quad+\left(P c_{T} K_{I}\left(\frac{\partial \lambda}{\partial t}\right)+P_{r} \frac{\partial \lambda}{\partial r}+P_{z} \frac{\partial \lambda}{\partial z}\right) \\
& \quad=\left(\left(\frac{i-q_{i}}{\rho}\right)\right)
\end{aligned}
$$



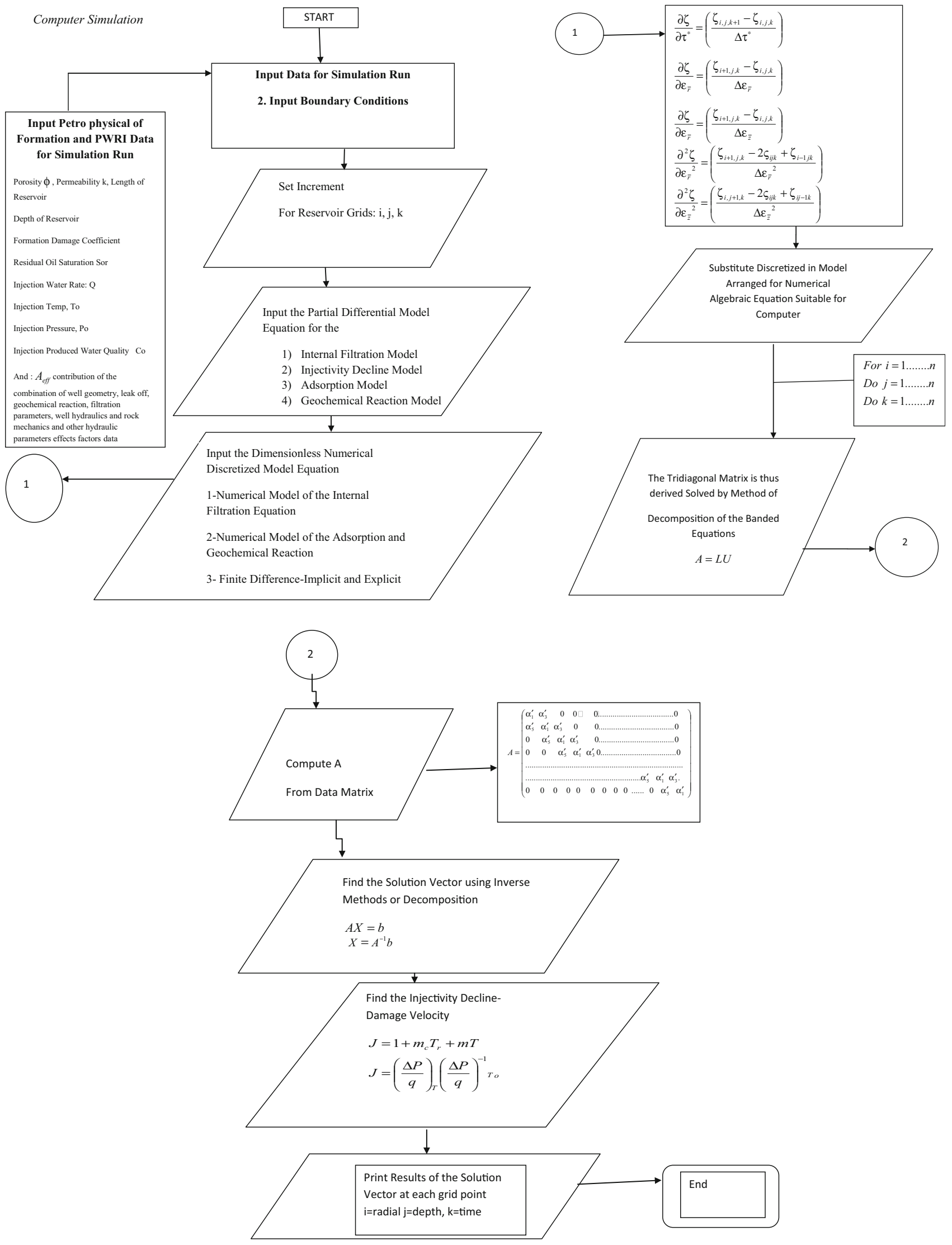

Fig. 4 Flow chart numerical simulation model 
where

$K_{I}=\frac{\partial \phi}{\partial \lambda}$

Measure the rate of flow ingress and egress

$$
\begin{aligned}
& \left(c_{T} \phi \frac{\partial P}{\partial t}+\lambda_{r} \frac{\partial P}{\partial r}+\lambda_{z} \frac{\partial P}{\partial z}+\frac{2 \lambda_{r} P_{r}}{r}\right) \\
& \quad+\left(P c_{T} K_{I}\left(\frac{\partial \lambda}{\partial t}\right)+P_{r} \frac{\partial \lambda}{\partial r}+P_{z} \frac{\partial \lambda}{\partial z}\right) \\
& \quad=b_{i}
\end{aligned}
$$

$b_{i}$ the permeability damage factor

$b_{i}=\left(\frac{i-q_{i}}{\rho}\right)$

The injectivity index model is defined as the flow rate per unity of the pressure drop between the injector and the reservoir. Injectivity decline is computed as in Eq. 69

$\prod=\frac{q(t)}{\Delta p(t)}$

The impedance is equal to the inverse of the dimensionless injectivity index

$J(T)=\frac{\prod(0)}{\prod(t)}=\frac{q_{o} \Delta p(T)}{\Delta p(0) q(T)}$

The impedance is a piecewise linear function of the dimensionless time for either deep bed filtration or external cake formation (Ajay and Sharman 2007) and now extended by a variable $R_{A T}(c)$ at transition point $T_{\mathrm{r}}$.

$J_{d}(T)=1+m T+R_{A T} T_{r} \quad$ for $T \leq T_{r}$

$J_{d}(T)=1+m T_{r}+m_{c}\left(T-T_{r}\right) \quad$ for $T>T_{r}$

$T_{r}>\frac{2 \alpha r_{w}}{\lambda C_{o} R_{c}^{2}}$

$m_{c}=\frac{k k_{\text {rowr }} \phi C_{o}}{k_{c}\left(1-\phi_{c}\right) X_{w}\left(-\operatorname{In}\left(X_{w}\right)\right)}$

The impedance slope $\mathrm{m}$ during the deep filtration is given by the formula below

$$
\begin{aligned}
m= & \left(\frac{\beta \phi c_{o}}{\operatorname{In} X_{w}}\right)\left(\lambda R_{c}\right)\left(\frac{1}{\sqrt{X_{w}}}\right)\left(-\exp \left(-\lambda\left(R_{C}-r_{w}\right)\right)\right. \\
& -\lambda R_{C} \exp \left(\lambda r_{w}\right) \int_{\lambda r_{w}} \frac{\exp (-u)}{u} \mathrm{~d} u
\end{aligned}
$$

where

$$
\begin{aligned}
& u=\lambda R_{c} \sqrt{X} \\
& X=\ell^{2}=\left(\frac{r}{R_{c}}\right)^{2}
\end{aligned}
$$

$X_{w}=\ell^{2}=\left(\frac{r_{w}}{R_{c}}\right)^{2}$

The slope $m_{c}$ during the external cake formation is: The computation of the velocity is given

$$
\begin{aligned}
& v_{r}=\frac{q_{r}}{2 \pi r h}=\left(\frac{K_{o r} K_{\sigma_{r}}}{\mu}\right) \int_{r_{w}}^{r_{e 1}} \frac{1}{r} \frac{d r P}{d r}+\int_{r_{e 1}}^{r_{e}} K_{o r} \frac{1}{r} \frac{d r P}{d r} \\
& v_{z}=\frac{q_{z}}{\pi r^{2} h}=\left(\frac{K_{o z} K_{\sigma_{z}}}{\mu}\right) \int_{r_{w}}^{r_{e 1}} \frac{d P}{d z}+\int_{r_{e 1}}^{r_{e}} K_{o z} \frac{d P}{d z} \\
& \int_{r_{w}}^{r_{e}}\left(\frac{q}{2 \pi h}\right) \frac{d r}{r}=\left(\frac{K_{o r}\left(1+K_{\sigma}\right)}{\mu}\right) \Delta P
\end{aligned}
$$

$\frac{\Delta P}{q}=\frac{\mu \operatorname{In}\left(\frac{r_{e}}{r_{w}}\right)}{2 \pi K_{o r}}+\frac{\mu \operatorname{In}\left(\frac{r_{e}}{r_{w}}\right)}{2 \pi K_{o r} K_{\sigma}}$

$\frac{\Delta P}{q}=\frac{1}{2 \pi K_{o r}}\left(1+\frac{1}{K_{\sigma}}\right)$

$\frac{\Delta P}{q}=\frac{1}{2 \pi K_{o r}}\left(1+\frac{1}{K_{\sigma}}\right)$

Total Impedance $=$ Damage Impedance

$$
+ \text { Undamaged Impedance }
$$

$\frac{\Delta P}{q}=\frac{\mu \operatorname{In}\left(\frac{r_{e}}{r_{w}}\right)}{2 \pi K_{o r}}+\frac{\mu}{2 \pi K_{o r}} K_{\sigma}^{\prime}$

$\frac{\Delta P}{q}=\frac{\mu}{2 \pi K_{o r}}\left(\operatorname{In}\left(\frac{r_{e}}{r_{w}}\right)+K_{\sigma}^{\prime}\right)$

Dimensionless form

$\frac{\left(\frac{\Delta P}{q}\right)_{T}}{\left(\frac{\Delta P_{O}}{q_{O}}\right)}=\frac{\left(\left(\operatorname{In}\left(\frac{r_{e}}{r_{w}}\right)+K_{\sigma}^{\prime}\right)\right)_{T}}{\left(\left(\operatorname{In}\left(\frac{r_{e}}{r_{w}}\right)_{\sigma}\right)\right)_{T}}$

The final form of injectivity model is presented in Eq. 89

$j=1+K_{\sigma}^{\prime}\left(\frac{1}{\operatorname{In}\left(\frac{r e}{r_{w}}\right)}\right)$

\section{Field data model analysis and computer simulation}

The studied field is a multi-reservoir, faulted anticline, heavy oil accumulation at a depth ranging from approximately 2200-2600 m subsea, in Campos Basin block BC4. Water depth within the areal extent of the field ranges 
from 1050 to $1300 \mathrm{~m}$. Studied field was developed as an all subsea well peripheral water flood project, with all injection below the various oil water contacts. The project uses vertical or deviated water injection wells and long, horizontal open-hole gravel pack production wells. At the time of this evaluation, a final decision has not been made regarding injection completion selection and also regarding whether produced water will be processed for overboard discharge or re-injected into the reservoir; therefore, this study will examine multiple completion geometries and the effects of alternative produced water strategies. The field data as reported in (Idialu 2014) were sourced in field report Wehunt (2002), Guo (2000), Meyer et al. 2003a, b.

\section{Modeling methods}

The simulation profiles for the water injection project are presented below and obtained from a Field Injection Study report Wehunt (2002). The values for all invariant simulation data are listed in 2 (Tables 1, 2, 3, 4). Additional information regarding what the various parameters are and how they function within the program is available from the program documentation. Details of the PWRI, well prognosis and simulation results for the effects of completion geometry, rock mechanics, filtration parameters, well hydraulics, leak off properties, operations, produced water re-injection parameters, reservoir properties are provided in "Appendix A". Details of the field report and data could be found in Wehunt (2002), Guo (2000), Meyer et al. (2003a, b). The reports highlight significance of (1) Completion geometry, (2) Rock mechanics (3) Filtration Properties (4) Total suspended solids (5) Filtration coefficient (6) Internal cake permeability damage factor (7) External filter cake permeability (8) Filter cake erosion ratio (9) Other leak off properties (10) Formation permeability (11) Injection fluid viscosity (12) compressibility (13) Aquifer oil saturation (14) Other assumptions (15) Boundary conditions, "ellipsoidal coupling, constant pressure B. C." was used for all runs except one. Ellipsoidal coupling, pseudo-steady state" was used for the other run. The fracture geometry was very insensitive to this parameter, and no plots are provided for this case. (16) Drainage Area; The BASE Case value was 1200 acres. Sensitivity cases were calculated for 750 acres and 2000 acres. The fracture geometry was very insensitive to this parameter, and no plots are provided for this case (17) Number of Fractures (18) Operations (19) Startup Procedure (20) Slurry Rate (21) Downtime (22) Wellbore Hydraulics in altering fracturing, permeability damage and injectivity. Results for this section are listed under the "Other Assumptions" category in Table 5 of their report.

\section{Results and discussions}

The results of model simulation based on the field data provided in "Field data model analysis and computer simulation" section were based on the field report and data obtained from Wehunt (2002), Guo (2000), Meyer et al. 2003a, b.

\section{Injector Performance and permeability damage as a function of aquifer integrity}

Figures 5 and 6 show injector performance with time related to fracturing hydraulics pressure and aquifer system. Figures 5 and 6 show field data simulation of a known field using Meyer fracturing simulator. Figures 5, 6, 7 and 8 show performance based on our software simulator in MATHLAB and COMSOL Multiphysics

Figures 5, 6, 7, 8 and 9 show the profile of permeability on both fracturing and filtration phenomena on the outlay in injector performance and concentration of cake build up. The profile decreased with time and increased uniformly with radial distance from produced water invasion zone. From the analysis of the results in the absence of particle deposition, low permeability formation was observed to be more likely fractured as the net fracturing pressure was observed to be inversely proportional to permeability, for a given injection rate. In addition, particle filtration and formation damage were governed by the interactions of particles in the injected water within the reservoir. In general, formation plugging is severe as the formation permeability decreased (Fig. 10).

Figure 11 shows profile of permeability and injectivity for 49 days for a particular field in Bakasap formation. The results were reported from the field and log data obtained and showed permeability damage with depth showing similar profile with Fig. 12, our simulated profile using COMSOL Multiphysics

Case 1: WID Simulation Data and Results

Figures 12, 13, 14 and 15 show fracture height with time and increase based on log data of PWRI case thermal and fractured profiles of decreased injector performance at different rates based on report Meyer et al. 2003a, b.

Figure 16 shows injectivity decline for different injection rates and shows a decrease with time and showing effect of fracturing pressure injector performance.

\section{Thermal and Pore Pressure Effects on Injectivity Performance}

Profiles in Figs. 17 and 18 show effect of thermal gradient in reservoir further to injectivity decline. Higher 


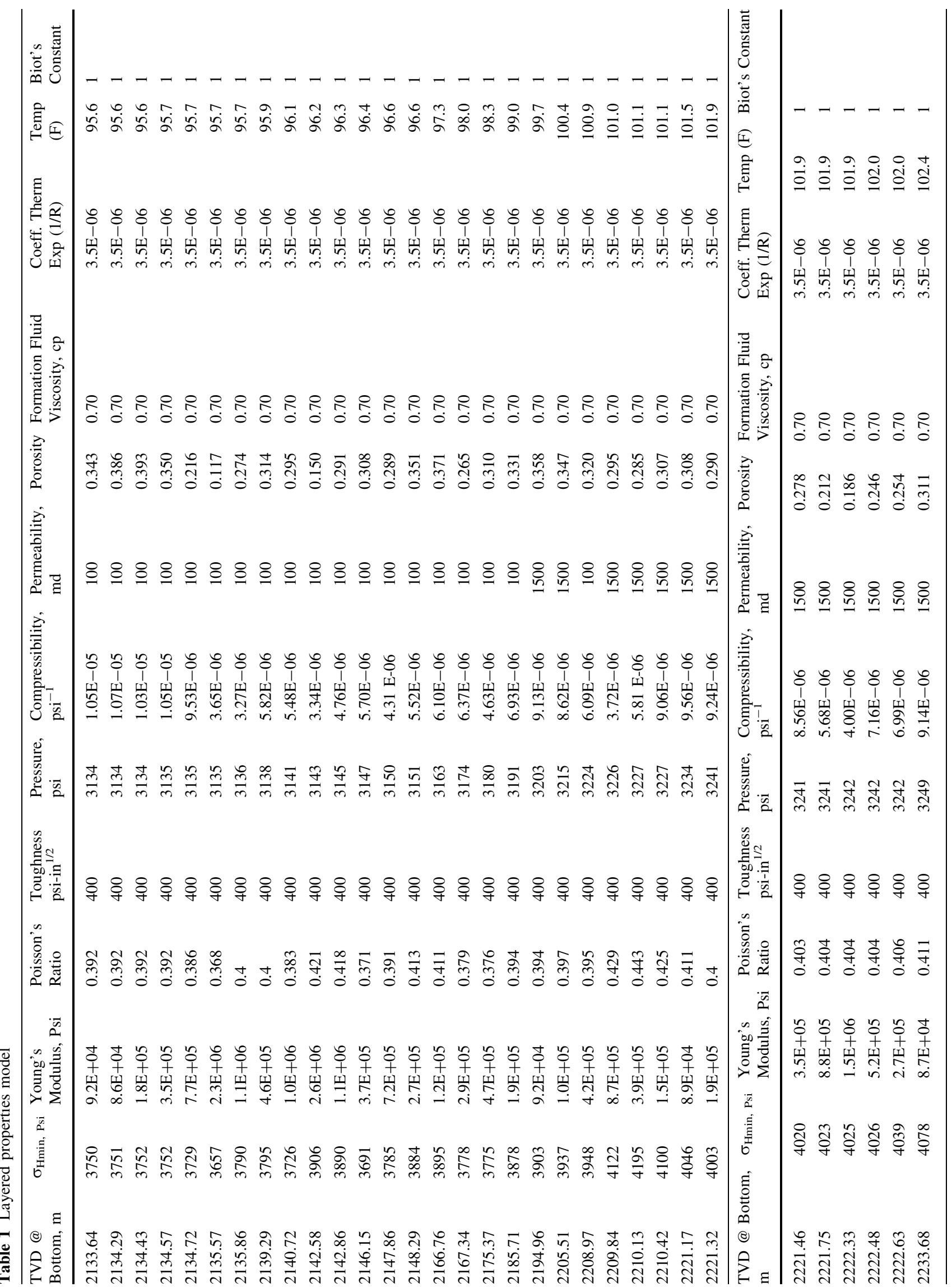




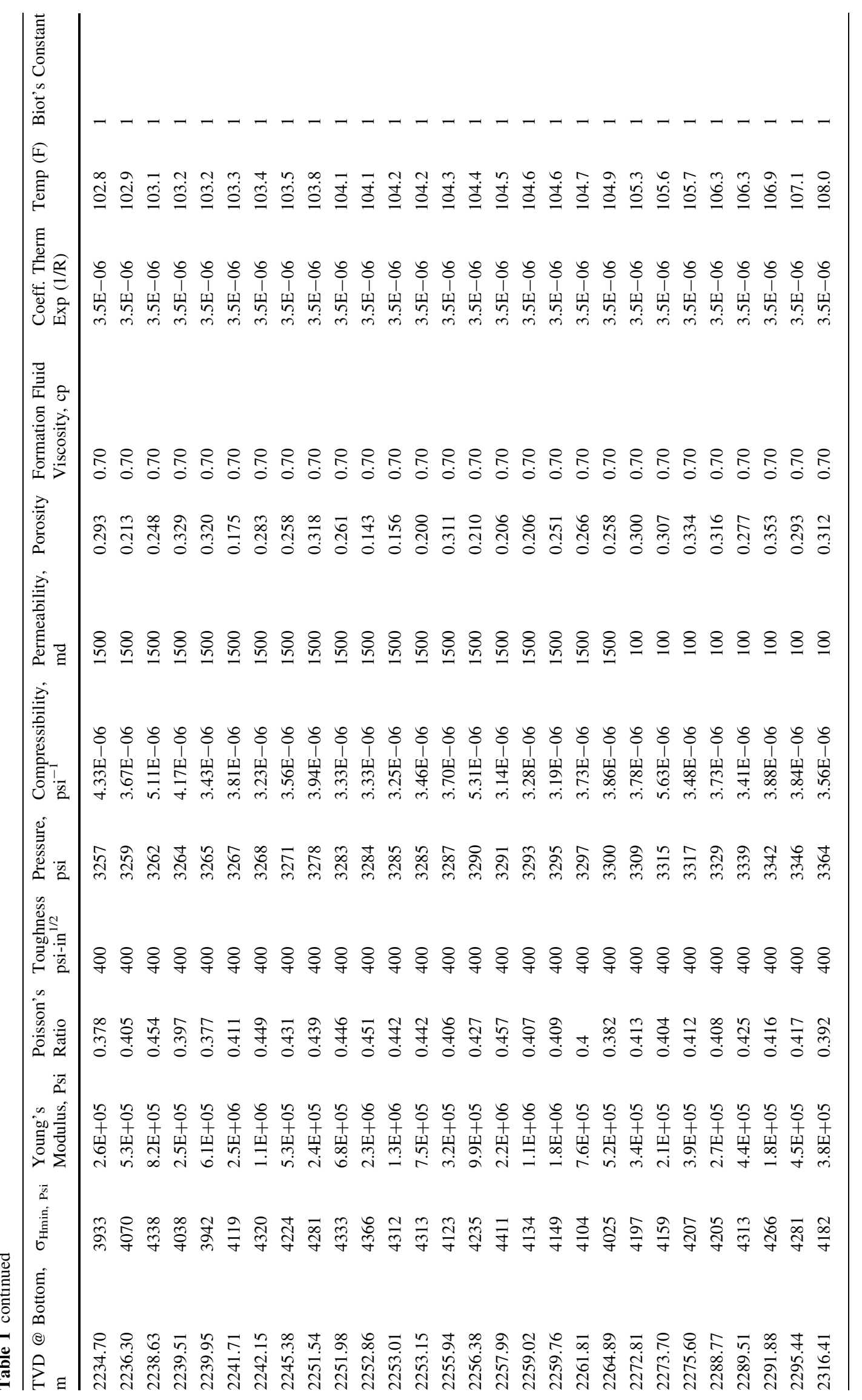


Table 2 Invariant simulation data

\begin{tabular}{|c|c|}
\hline Description & Value \\
\hline Fluid loss model & $\begin{array}{l}\text { Dynamic, calculate fracture } \\
\text { skin, and include fluid loss } \\
\text { history }\end{array}$ \\
\hline Fracture geometry & 3-Dimensional \\
\hline Flow back & Off \\
\hline Simulate to closure & On \\
\hline Fracture fluid gradient & Include \\
\hline Propagation parameters & Default Growth $(+,-)$ \\
\hline Fracture initiation interval & Minimum stress interval \\
\hline Fracture friction model & On, with $a=24$ and $b=1$ \\
\hline Wall roughness & Off \\
\hline Tip effects & Off \\
\hline Flow path & Tubing \\
\hline Surface line volume & $0 \mathrm{bbls}$ \\
\hline Depth & $2210 \mathrm{~m} \mathrm{MD}$ \\
\hline Maximum BHTP & $6000 \mathrm{psi}$ \\
\hline Fixed depth & MD \\
\hline Calculated (TVD or Angle) & Angle \\
\hline Deviation survey & $\begin{array}{l}\text { Based on 3-TXCO-3DA, } \\
\text { MD:TVD, 22:22, } \\
\text { 1332:1332, 2143.45:2133.6, } \\
\text { 2334.98:2316.48, } \\
\text { 2506:2473 }\end{array}$ \\
\hline Casing & $9-5 / 8^{\prime \prime} 47 \#$ set at $2506 \mathrm{~m} \mathrm{MD}$ \\
\hline Tubing & $5-1 / 2^{\prime \prime} 20 \#$ set at $2180 \mathrm{~m} \mathrm{MD}$ \\
\hline Downhole flow restrictions & None \\
\hline Perforation size & $0.5^{\prime \prime}$ \\
\hline Additional near wellbore friction & None \\
\hline Schedule type & Bottom hole \\
\hline Stage type & Pad \\
\hline Injection fluid type & KCL2 \\
\hline Injected fluid type & Water \\
\hline Reservoir lithology type & Sandstone \\
\hline In situ fluid & Water \\
\hline Non-pay permeability & $100 \mathrm{md}$ \\
\hline In situ fluid viscosity & $0.7 \mathrm{cp}$ \\
\hline Irreducible water saturation & 0 \\
\hline $\begin{array}{l}\text { Deposited concentration ratio after } \\
\text { transition }\end{array}$ & 0.5 \\
\hline Permeability damage power & 0.1 \\
\hline Cake porosity & 0.25 \\
\hline $\begin{array}{l}\text { Fractional deposition of TSS } \\
\text { building cake }\end{array}$ & 0.5 \\
\hline Cake build & 1 \\
\hline Cake erosion & 1 \\
\hline
\end{tabular}

Table 3 Zone data

\begin{tabular}{llllll}
\hline & \multicolumn{4}{l}{ TVD Depths from Rig Floor, $\mathrm{m}$} \\
\cline { 2 - 3 } & \multicolumn{2}{l}{ Perforations } & & \multicolumn{2}{l}{ Zone } \\
\cline { 2 - 3 } \cline { 5 - 6 } Zone name & Top & Bottom & & Top & Bottom \\
\hline Top & 2181.10 & 2187.02 & & 2174.24 & 2193.24 \\
Upper & 2198.88 & 2204.83 & & 2196.55 & 2209.63 \\
Lower & 2217.74 & 2223.72 & & 2209.63 & 2231.36 \\
Bottom & 2237.71 & 2243.72 & & 2231.36 & 2249.97 \\
All & 2181.10 & 2243.72 & 2175.19 & 2249.75 \\
\hline
\end{tabular}

Table 4 Seawater versus PWRI case data

\begin{tabular}{lll}
\hline Variable & $\begin{array}{l}\text { Seawater } \\
\text { cases }\end{array}$ & $\begin{array}{l}\text { PWRI } \\
\text { cases }\end{array}$ \\
\hline $\begin{array}{l}\text { Injected fluid temperature, F } \\
\text { Injected fluid viscosity, cp }\end{array}$ & 60 & 120 \\
$\begin{array}{l}\text { Internal filter cake permeability damage } \\
\text { ratio }\end{array}$ & 1.12 & 0.60 \\
\begin{tabular}{l} 
External filter cake permeability, mD \\
\hline
\end{tabular} & 0.0100 & 400 \\
\hline
\end{tabular}

temperature favors the reduction of particle deposition in reservoir. This validates established technique in the industry called stimulation whereby heat injected into the reservoir clean pore spaces of deposition. The simulation profiles showed that fracture gradient was more likely to be influenced by pore pressure and temperature changes. As cooler injection fluids reduce temperature, the rock becomes more brittle, strongly dependent on Young's Modulus of elasticity. Injection flow rate is an important parameter in permeability impairment. The higher the linear velocity, the greater the depth of particle penetration. Smaller velocities and larger particle concentration results in larger permeability declines and thus greater decline is experienced. From the graph above, it is seen that the increase in the fluid flow rate results in the internal cake forming faster.

The results of model simulation based on the field data provided in "Field data model analysis and computer simulation" section were based on the field report and data obtained from "In Situ Stress and Wellbore Stability Field, Brazil," CPDEP Phase 2 report DR-AP-RP-021,209 simulation as reported by GeoMechanics International, Inc., Guo (2000), Meyer et al. 2003a, b. 


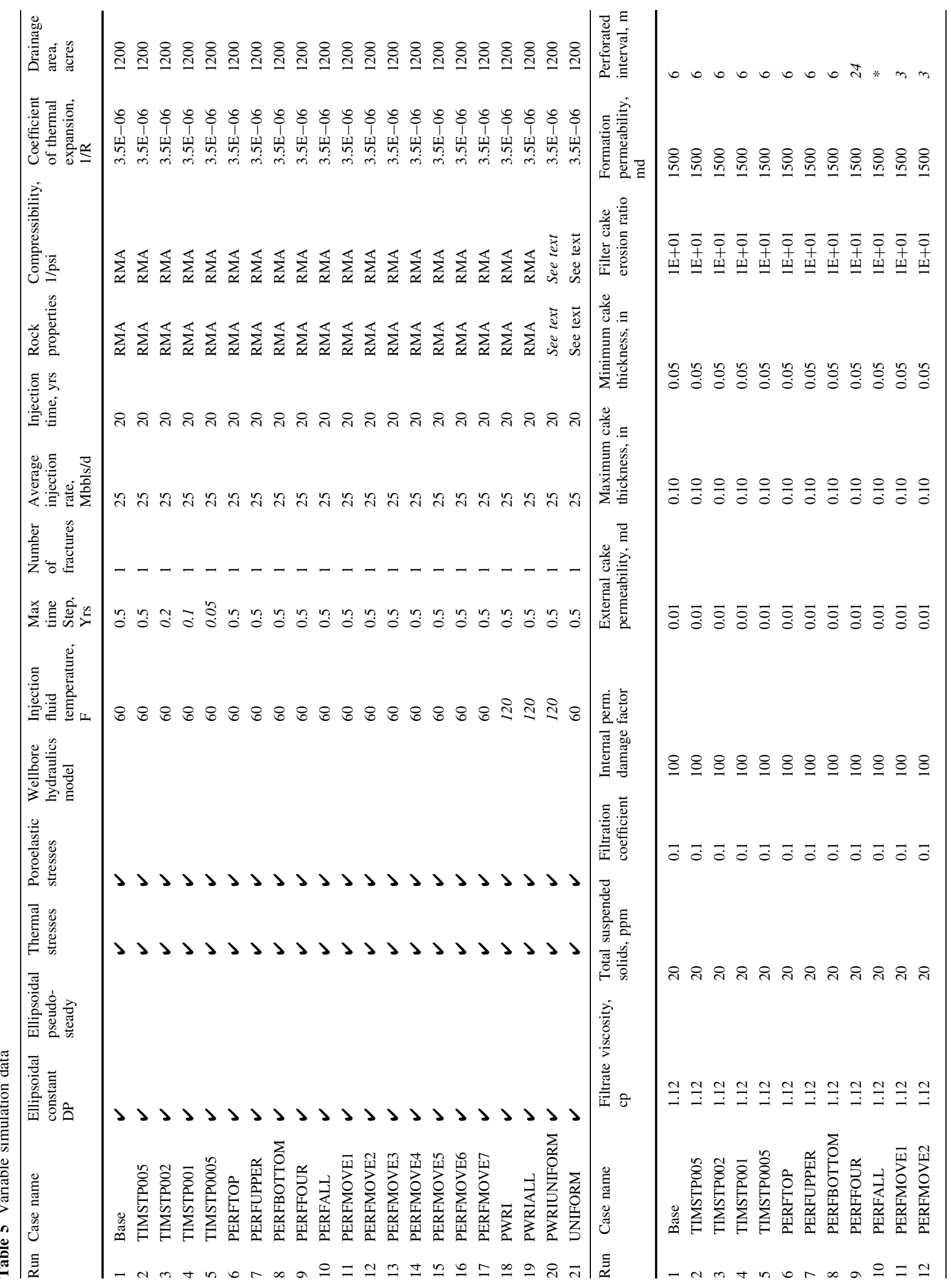




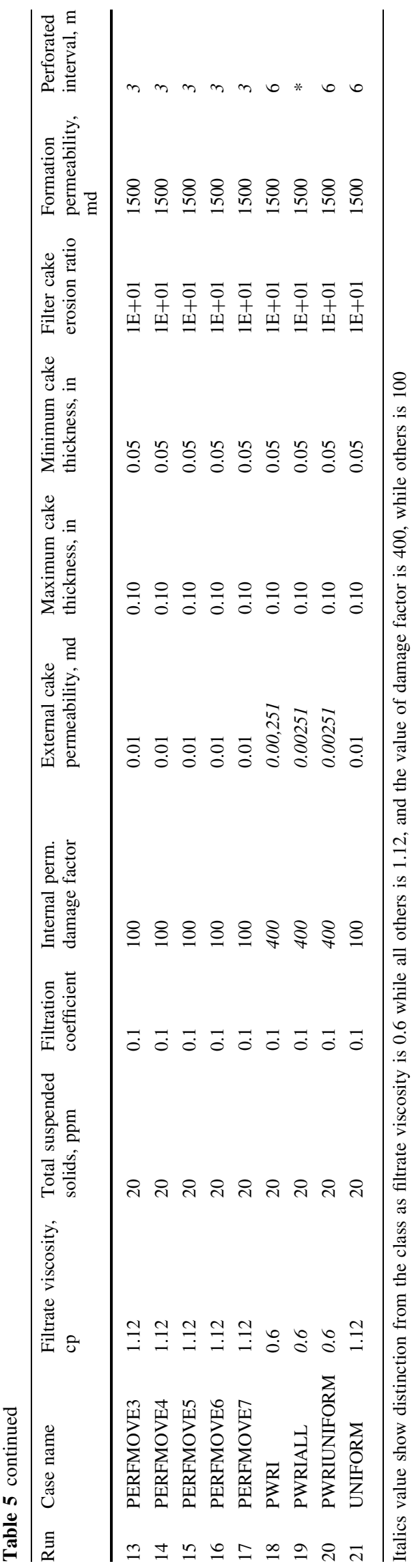

Figure 19 shows Bekasap Formation of the Kotabatak field as well as produced water from the Bekasap formation from other fields in the areas such as Kasikan, Lindai, Langgak, Petapahan; that the higher pressures seen are a good indication of the maximum pressure expected before fracture extension occurs, in this case about 2900 psi. Results of Fig. 19 show that lower pressures seen that either have low injection rates or have recently had a fracture extension. In either case, they are an indication of what the reservoir pressure would be (about $2000 \mathrm{psi}$ ). Similarly, this is the pressure ultimately seen by a hydraulic fracture conducted on a producing well.

Figure 20 shows the output of the WID (water injectivity decline) simulator, using data input to roughly simulate a Kotabatak injector, for a case with a 20 -foot fracture. Note that injection proceeds steadily for about a year and then suddenly drops. This corresponds to the behavior seen in Fig. 6 for Well 190, where a pressure spike occurs about once a year. Injection rate climbs slightly for a majority of that year, followed by a swift decline in injection and increase in pressure. This cycle has been repeated several times, which can be interpreted as fracture growth/extension occurring about once a year, at least for this well.

\section{Injectivity performance and permeability damage on rock properties}

Profiles in Fig. 21 show that well injectivity varies during water injection basically due to two competitive factors: formation damage by the suspended particles which results into deposition and thus injectivity decline. As shown in Figure 30, for different injection rates, injectivity decline exponentially decreases with time and increases as the

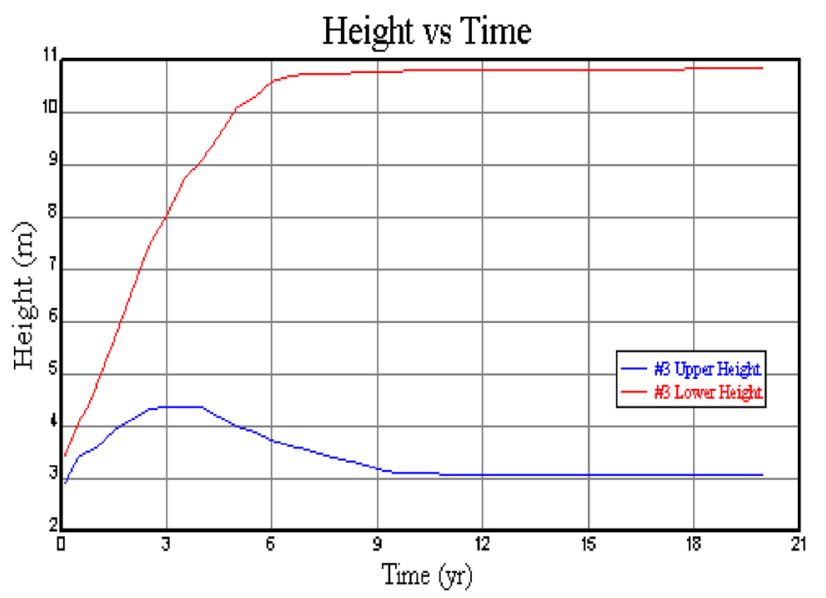

Fig. 5 Height of fracturing with time 

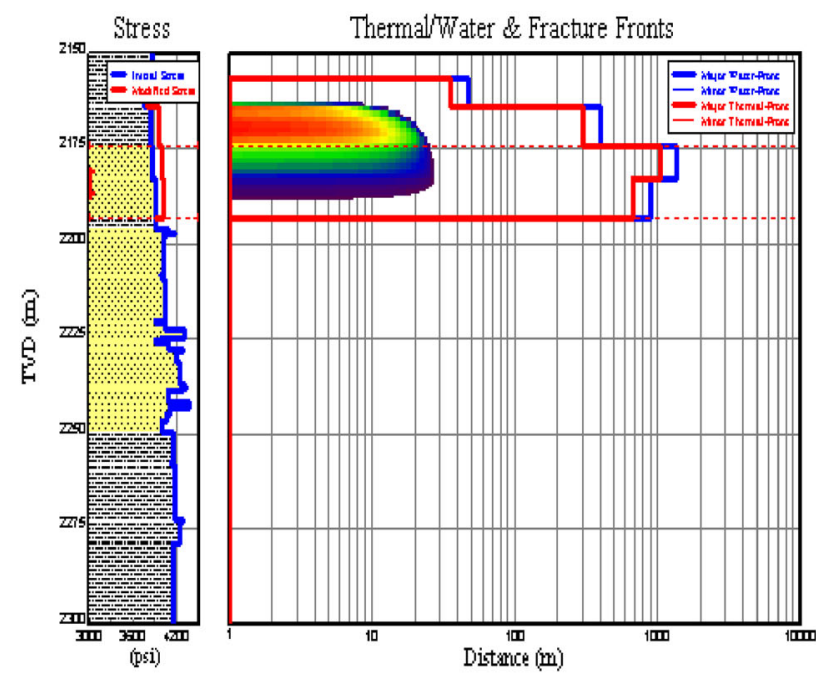

Fig. 6 BASE case fracture height versus time

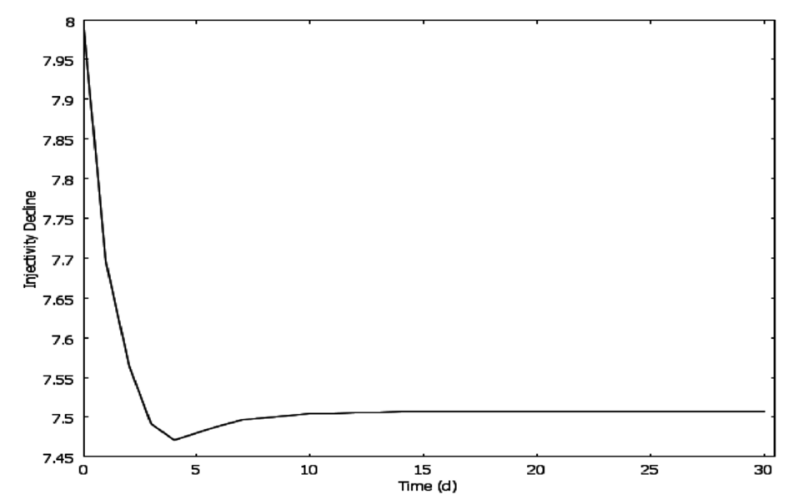

Fig. 7 Injector performance with time

produced water injection rates increase. Injectivity index decline decreases with damaged factor exponentially are plotted in Fig. 22. Injectivity decreases from 1.126 to zero when damaged factor is 1.0 indicating the effects of cake deposits in pore blocking and permeability damage. The injectivity decline experienced in the reservoir has been linked to the volume of oil produced. From the graph, it is observed that the injectivity decline experienced increases as the production rate reduces. This is better explained by suggesting that mobility ratio, voidage factor and reservoir permeability has a profound influence on both fracturing and filtration phenomena. Even in the absence of particle deposition, low permeability formation is more likely to be fractured as the net fracturing pressure is inversely proportional to permeability, for a given injection rate. In addition, particle filtration and formation damage are governed by the interactions of particles in the injected water with the reservoir rock. In general, formation
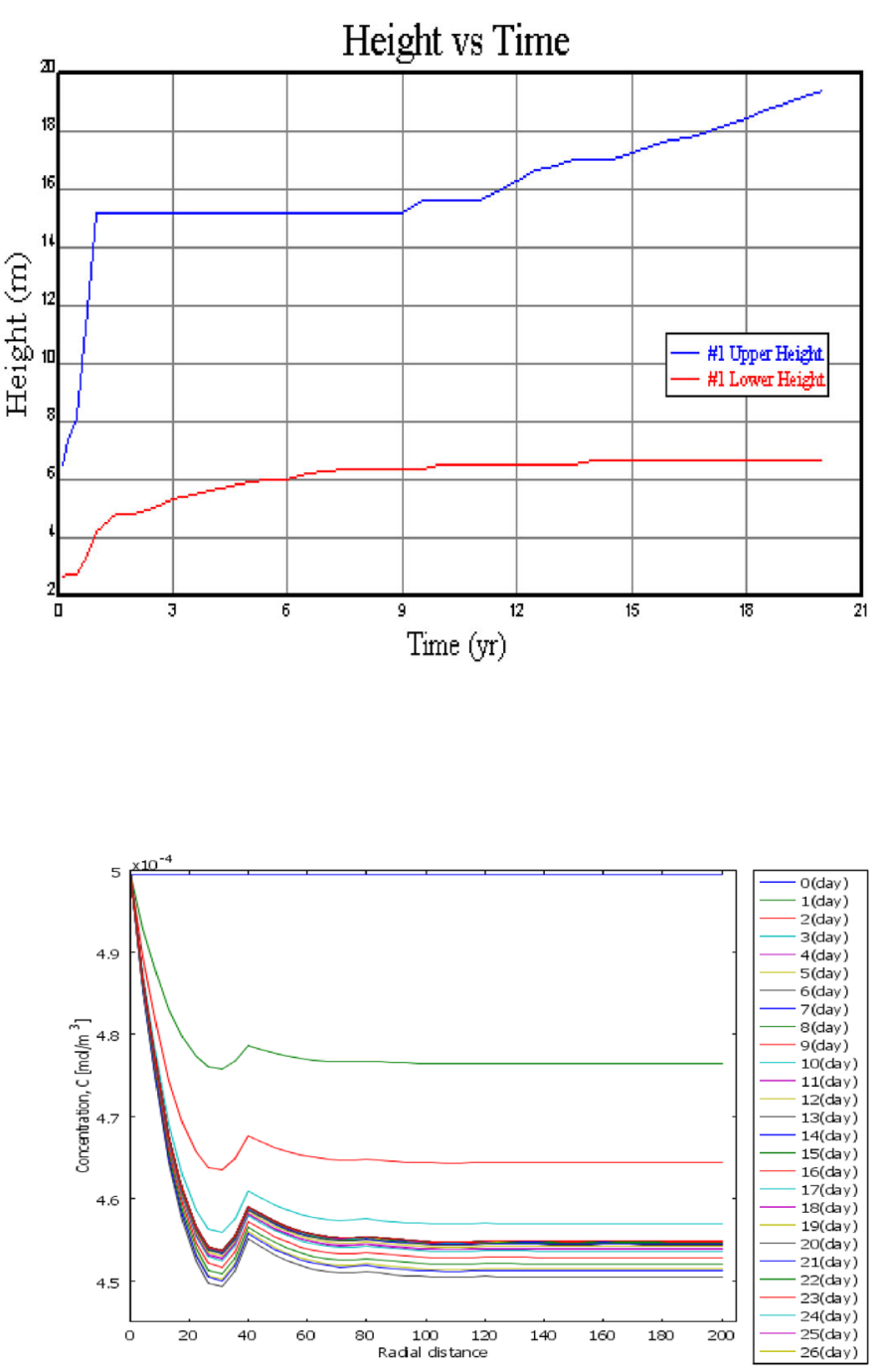

Fig. 8 Concentration profiles with time

plugging will be more severe as the formation permeability decreases. It should be noted here that the formation permeability is directly dependent upon the formation grain size (dg). Particle deposition around the wellbore and the fracture face, modeled using filtration theory. This influence is via an increase in injection pressure due to additional skin resistance across the face of the fracture or near wellbore. This additional flow resistance is due to combination of internal and external cakes. The pressure increase due to skin resistance is inversely proportional to the area of fracture face with differing particle size, we find out that (1) overall damage is related to the mean pore throat size (2) the pore damage with $0-3$ microns exhibit damage throughout the entire reservoir length (3) as particle size increase, the damage is gradually shifted toward the injection end of the pore and to an external cake. Particles of sizes ranging from $0.05-7$ cause formation damage. The 

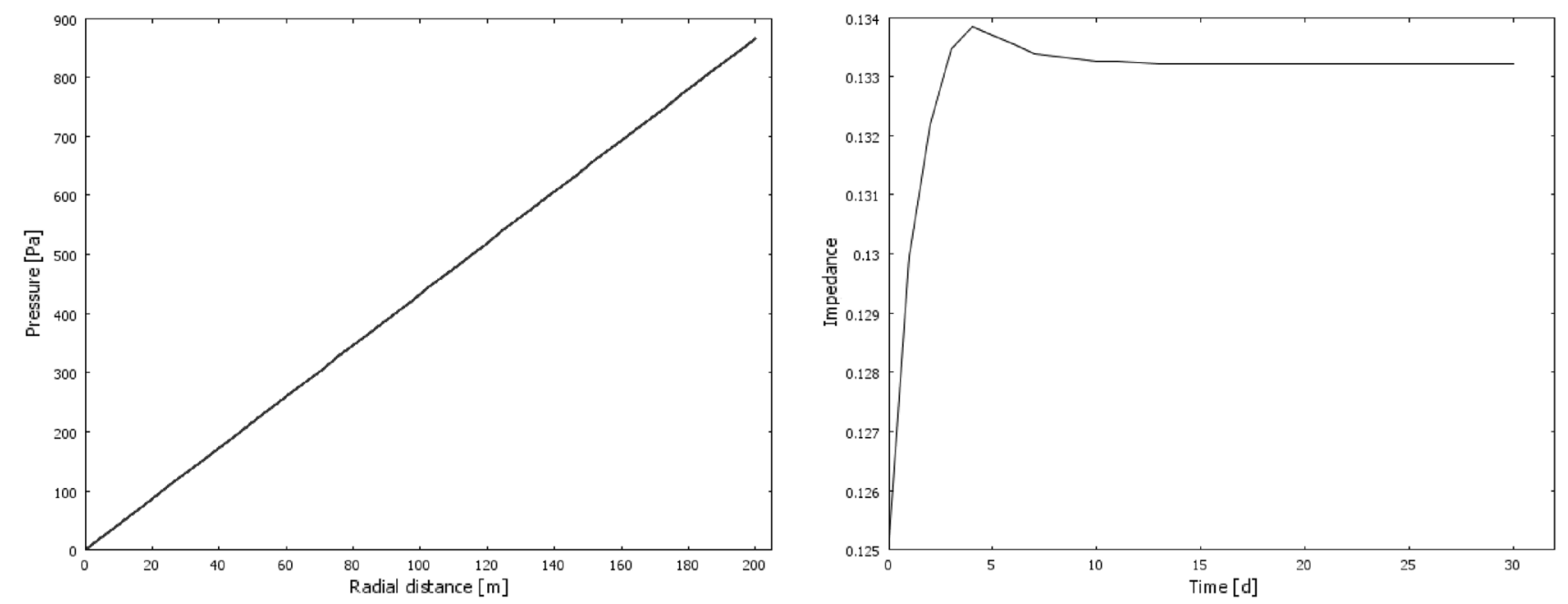

Fig. 9 Plot of fracturing pressure on impedance with time
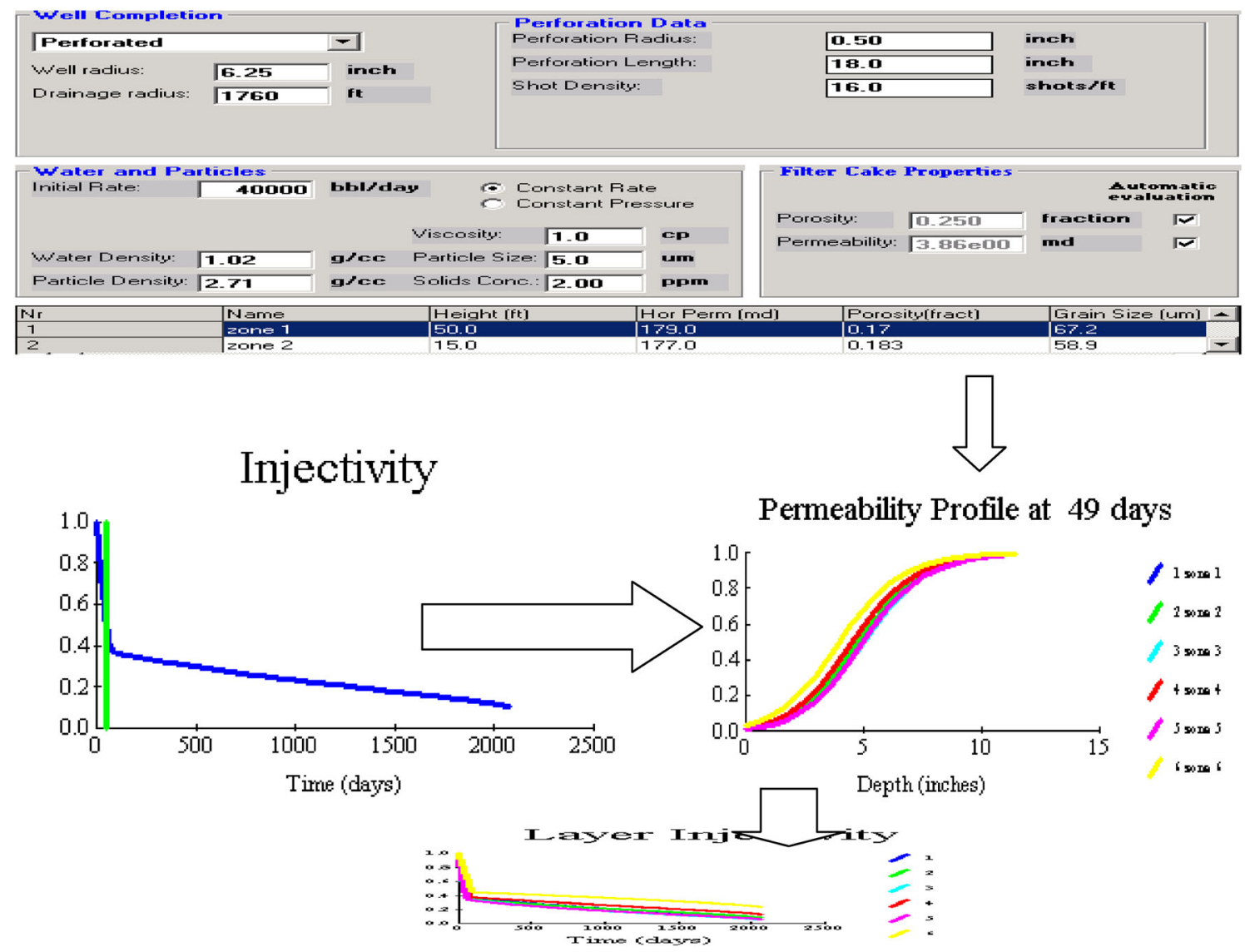

Fig. 10 Profile of injectivity decline of produced water from the Bakasap formation. source: Energy Tech Co, Houston, Texas and Department of Petroleum Resources [Nigeria] as reported by Idialu (2014)

larger particles cause a rapid decline in permeability with the damage region being shallow. Smaller particles enter the core and cause a gradual permeability decline.

Figures 23 and 24 injector performance profiles showed the effect of ratio of particle size to reservoir pore size on injectivity decline as the ratio increases, injectivity decline decreases as well, and all injectivity decline decreases with time. When suspended particles in a carrier fluid are flowed through a porous medium, the operative plugging mechanism depends on the 
Fig. 11 Permeability damage with depth and time

Fig. 12 Field Studied case fracture height versus time
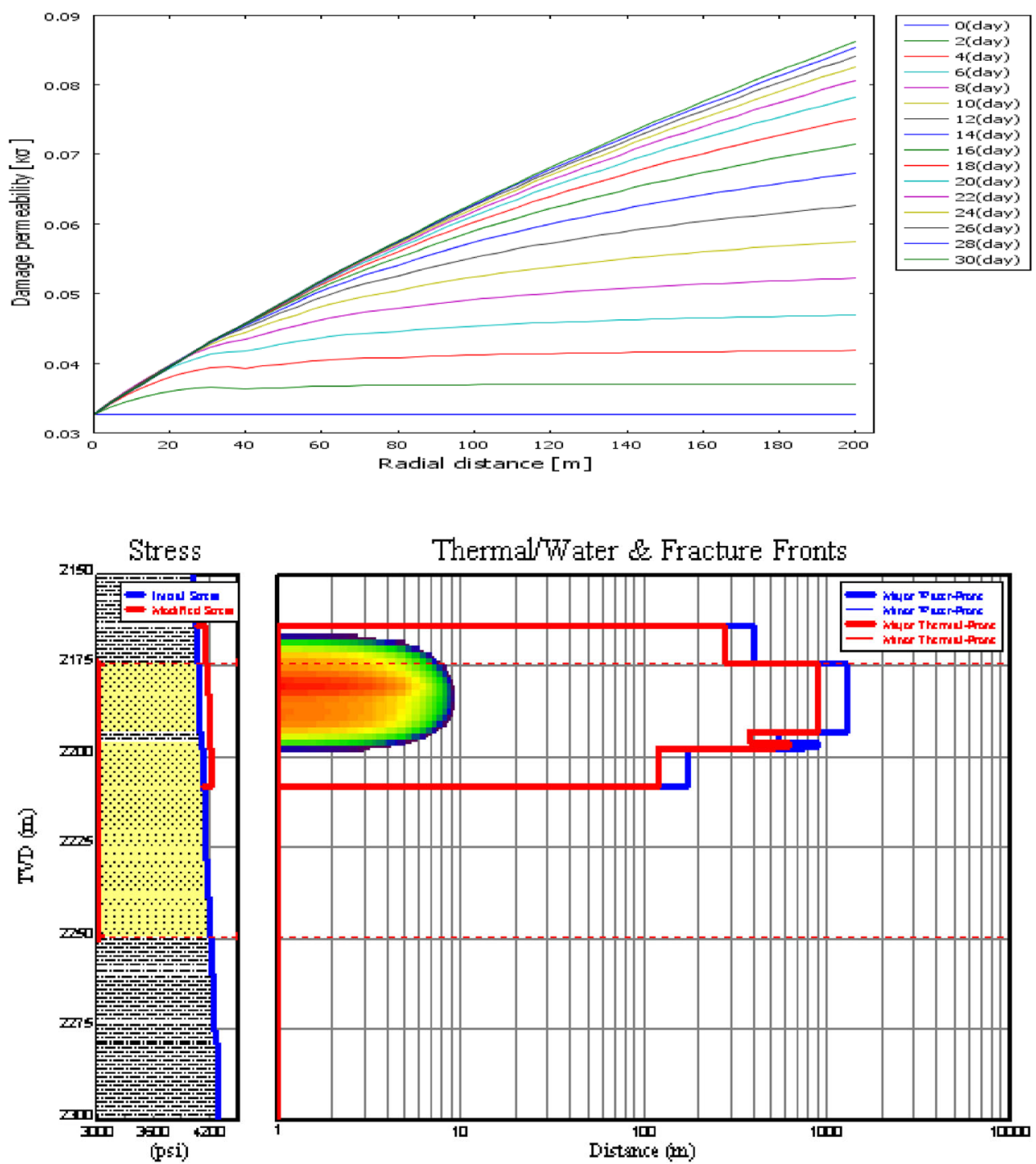

Thermal/Water \& Fracture Fronts

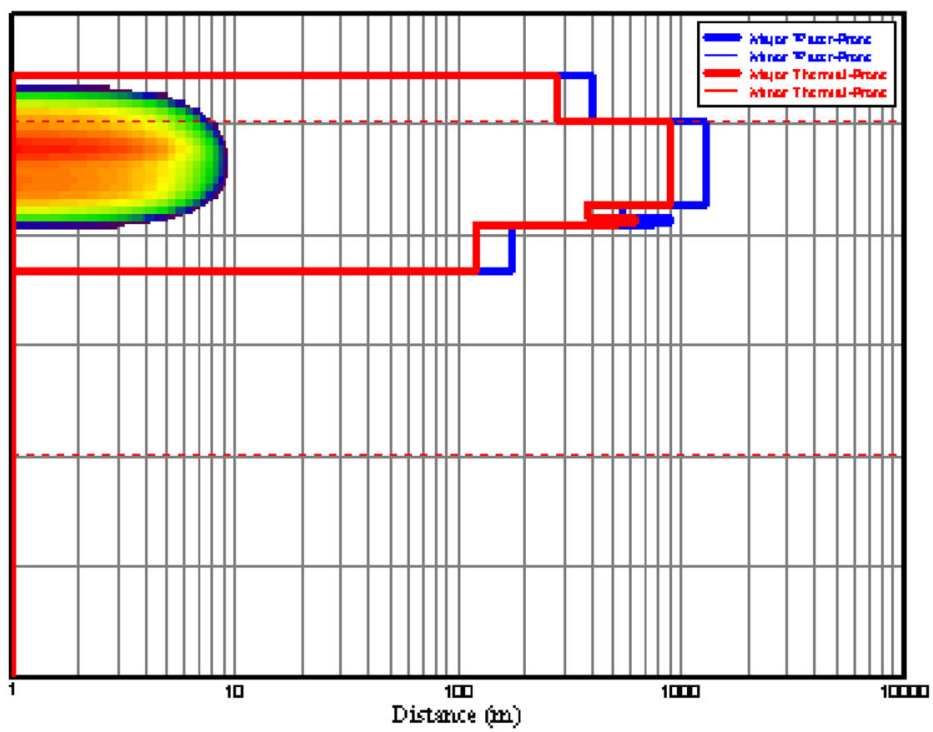

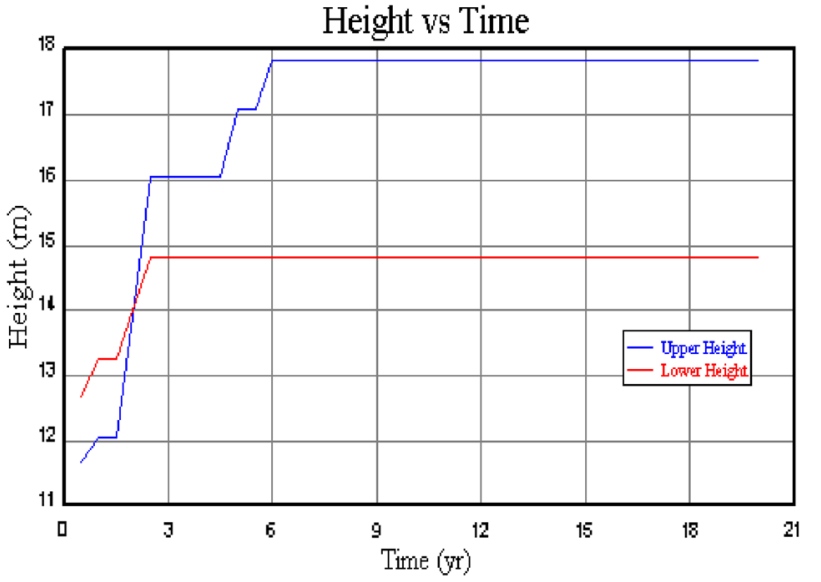

Fig. 13 Field Studied case thermal and fracture profiles characteristics of the particle, the characteristics of the formation, and the nature of the interaction between the particle and the various reservoir materials. With differing particle size, we find out that (1) overall damage is related to the mean pore throat size (2) the pore damage with 0-3 microns exhibit damage throughout the entire reservoir length (3) as particle size increases, the damage is gradually shifted toward the injection end of the pore and to an external cake. Particles of sizes ranging from 0.05-7 cause formation damage. The larger particles cause a rapid decline in permeability with the damage region being shallow. Smaller particles enter the core and cause a gradual permeability decline. The particle/pore size ratio is the most important parameter in the filtration process. It can be seen that the larger the particle/pore size ratios tend to cause rapid, but shallow damage. As shown from the graph, varying the damage 
Fig. 14 Thermal/water and fractured fronts
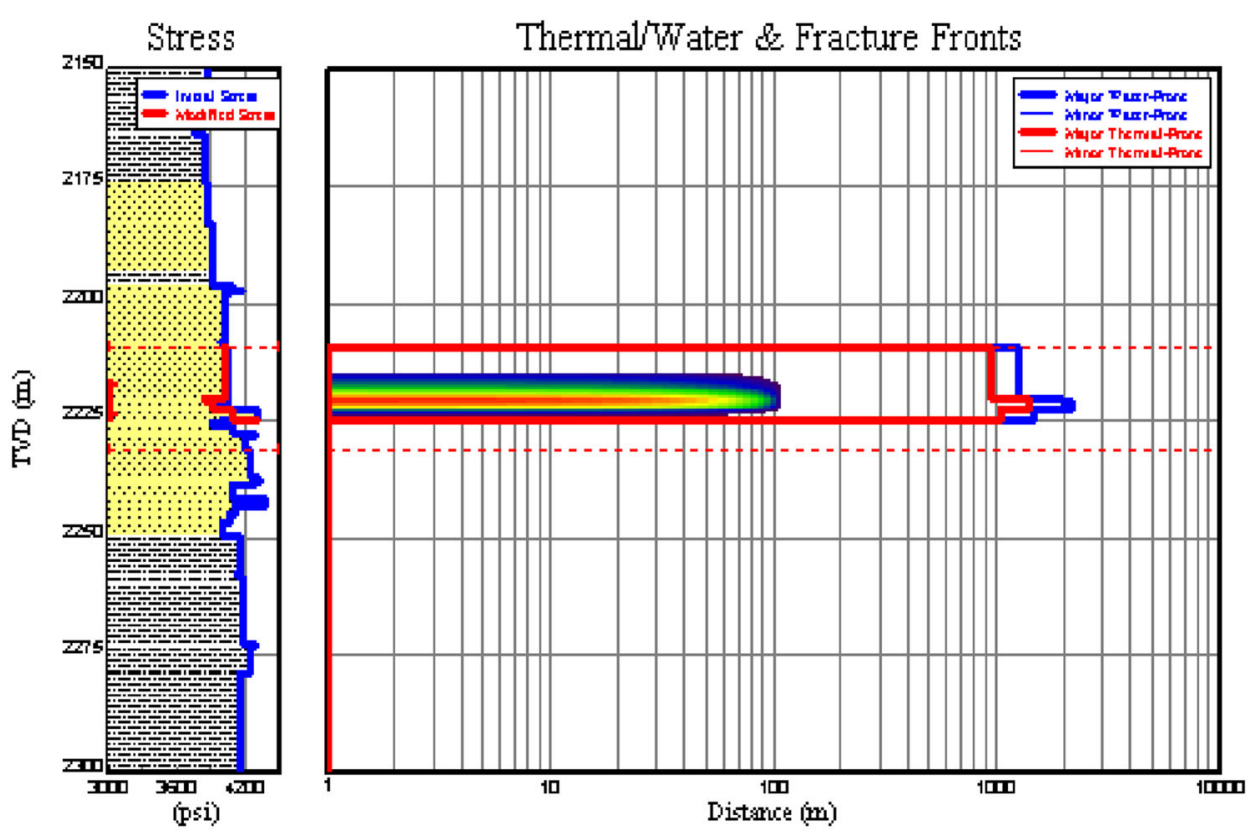

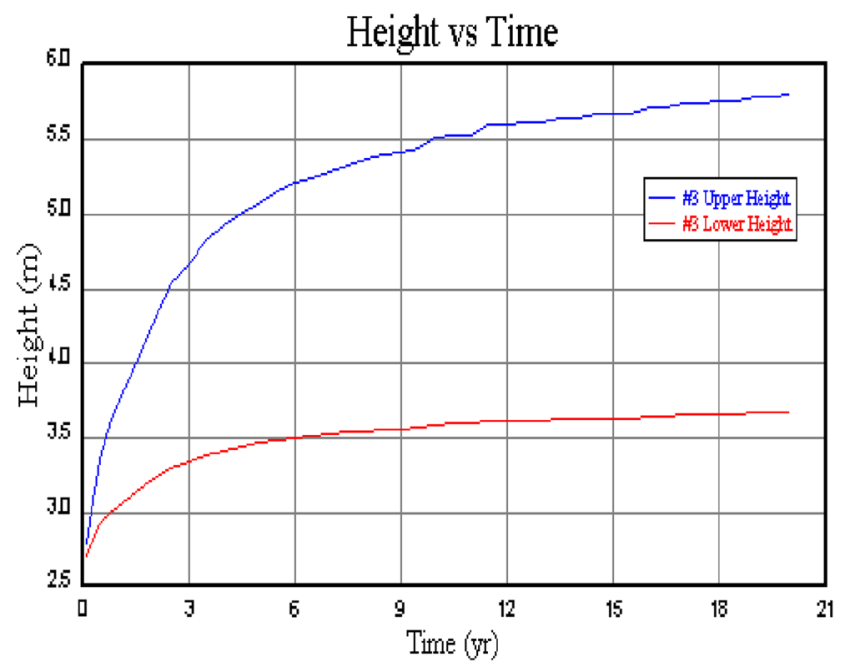

Fig. 15 Fracture height versus time factor used for the simulation would have little or no effect on the outcome of the simulation. The injectivity decline experienced even with these varying factors and days showed that the decline has very little dependence on these factors.

\section{Conclusion}

An improved internal filtration model incorporating the effect of adsorption kinetics, geochemical reaction and hydrodynamics, well hydraulics and aquifer integrity residual oil mobility and correction for well completion geometry and rock mechanics formation damage coefficient introduced as $R_{\mathrm{AT}}$ variables that highlights the contribution of the combination of well geometry, leak off,
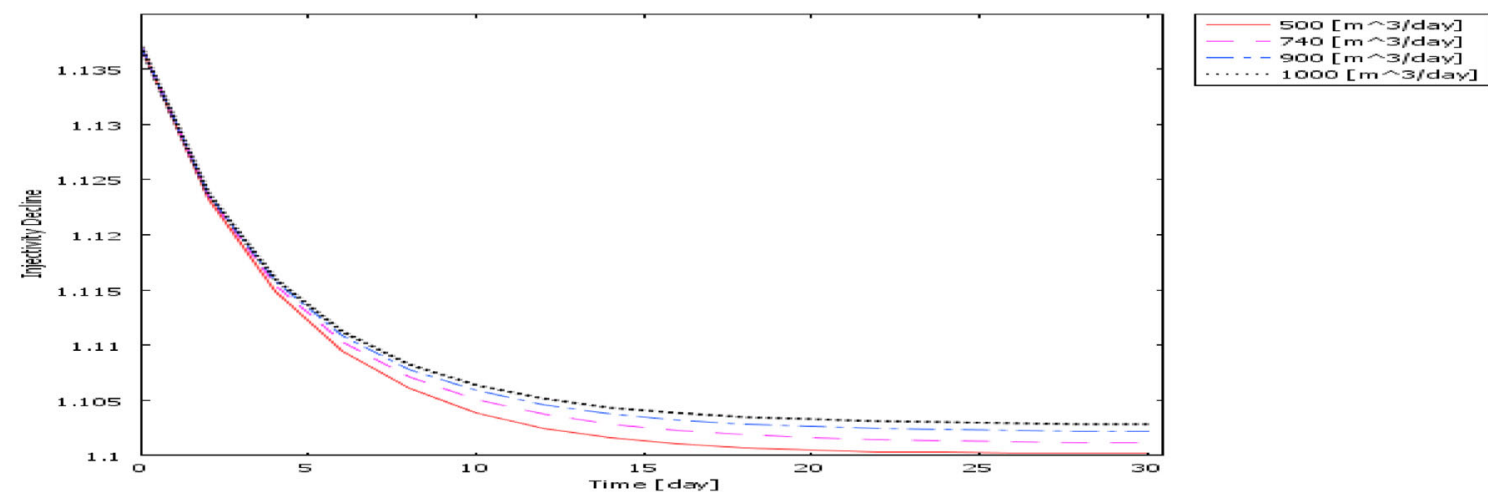

Fig. 16 Injectivity decline with time for different injection rates 
Fig. 17 Injectivity with time at different rates

Fig. 18 Injectivity with time at different temperature
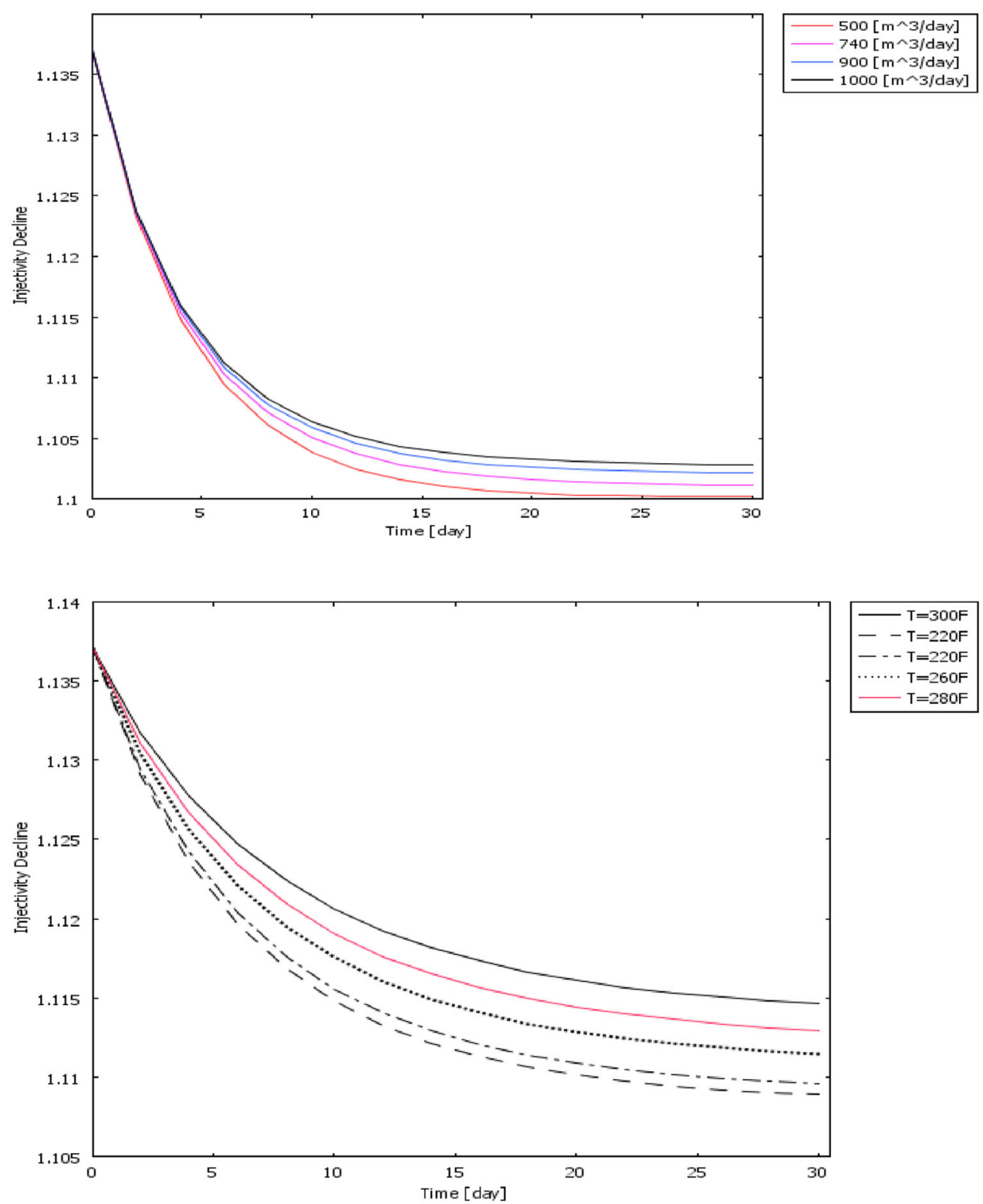

geochemical reaction, filtration parameters, well hydraulics and rock mechanics and other hydraulic parameters effects factors. The model injectivity and fracturing was solved using the finite element method simulated in COMSOL Multiphysics Software. To simulate the model, well-known implicit finite difference discretization scheme was employed to the improvements in advection-dispersiongeochemical reaction process incorporating the variable $R_{\mathrm{AT}}$ in a dimensionless time constants. The attendant banded linear systems of equations were solved in MATHLAB environment using decomposition approach. Using preliminary field data obtained from re-injection sites in the Injection Field Project, our simulation showed that permeability decline is exponential function in time of $R_{\mathrm{AT}}$ factors signifying of aquifer integrity, rock mechanics properties, thermal stress, particle to grain ratio, retention kinetics, filtration parameters, well hydraulics, and produced water quality in $R_{\mathrm{AT}}$ function alters permeability damage, fracturing, cake formation and injectivity decline in an improved robust improved internal filtration-hydraulic model. However, at a specific length in the aquifer, the concentration profile of the active specie follows an exponential distribution in time. Meanwhile, injectivity decline decreases exponentially with radial distance in the aquifer. Clearly, injectivity decline is a function of fracturing mechanics for injector performance and cake deposition resulting in permeability damage $\mathrm{g}$ from an adsorption coupled filtration scheme. In this regard, it is established that the transition time $t_{r}$ to cake nucleation and growth is a consequence aquifer capacity, filtration 


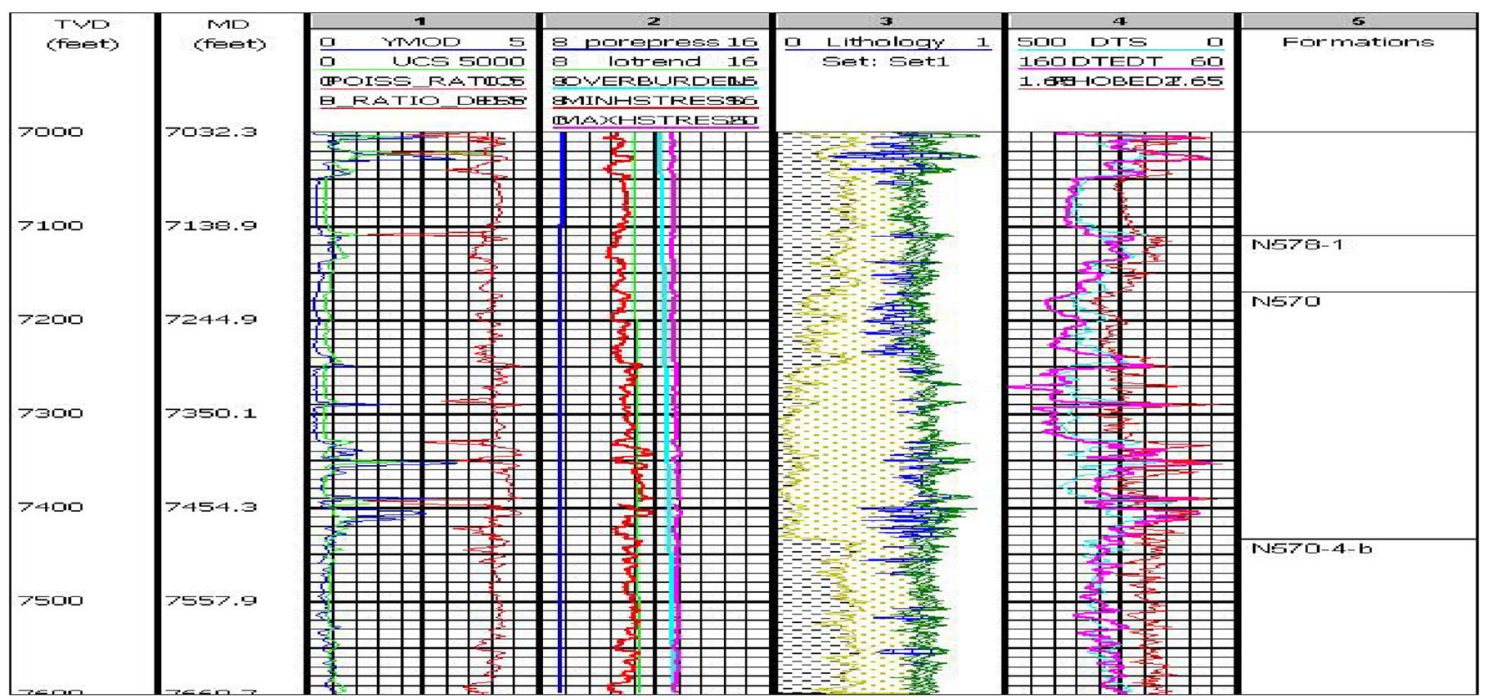

Fig. 19 PERFTOP case thermal and fracture profiles
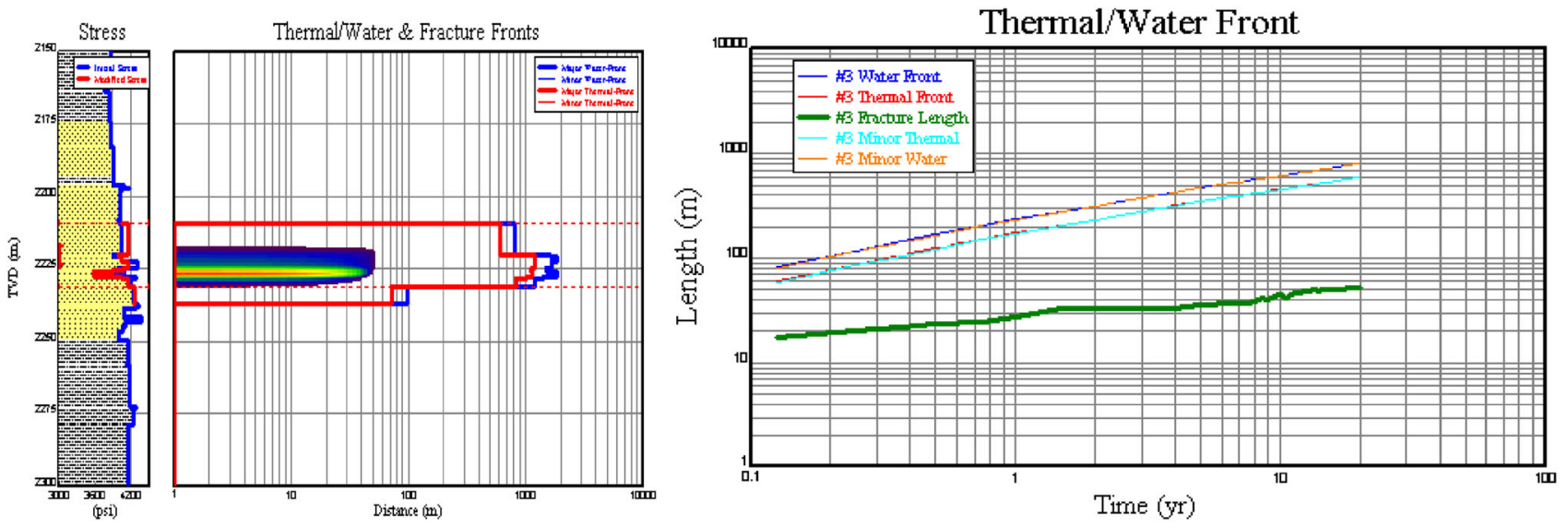

Fig. 20 PERFTOP case fracture height versus time
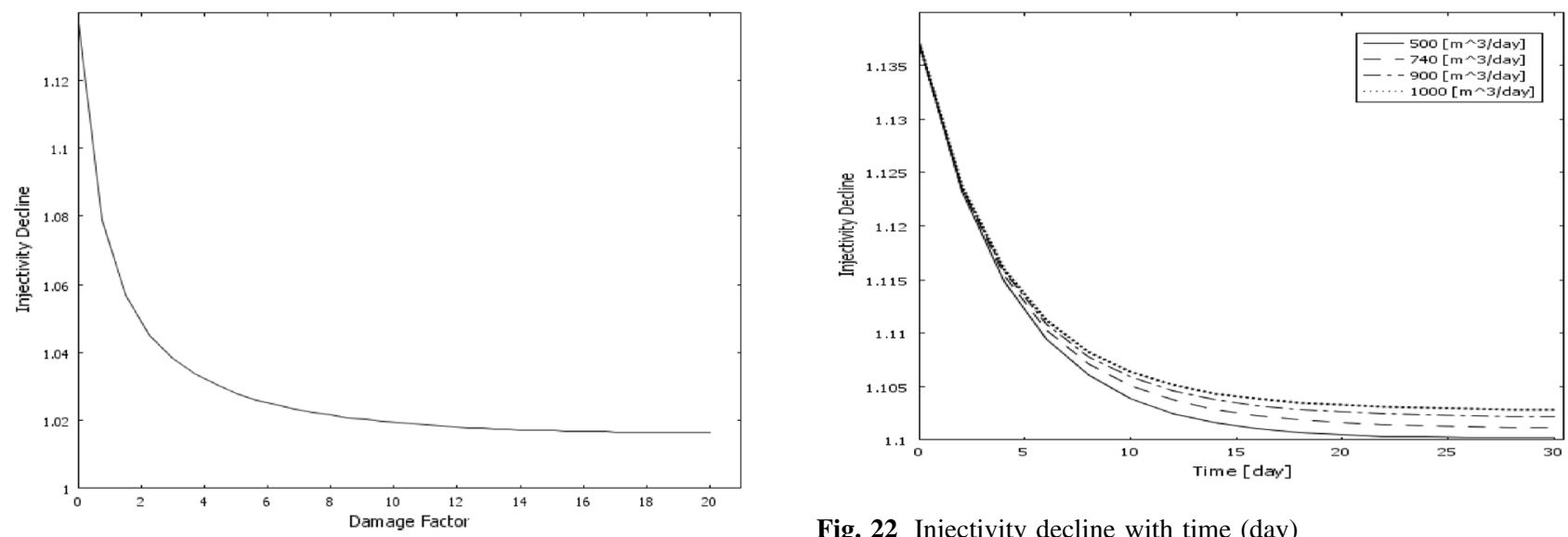

Fig. 22 Injectivity decline with time (day)

Fig. 21 Injectivity decline with damage factor 
Fig. 23 Injectivity decline with damage factor in days

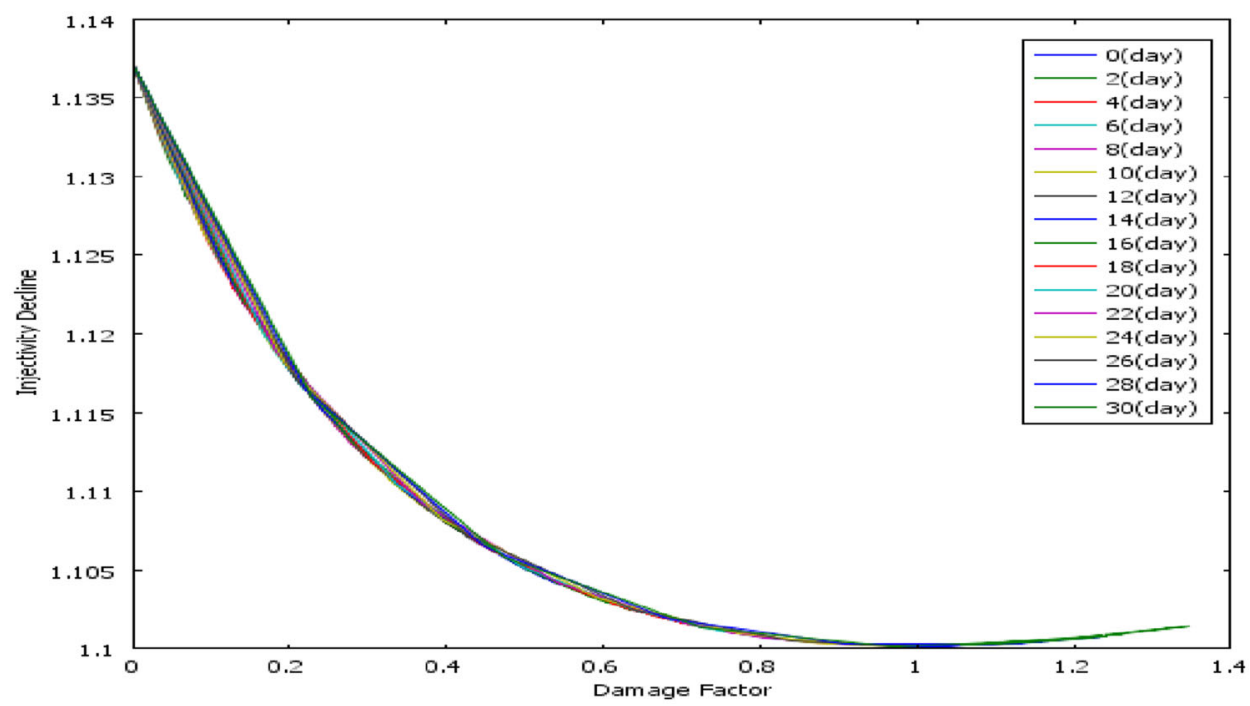

Fig. 24 Injectivity decline with time for different damage factors

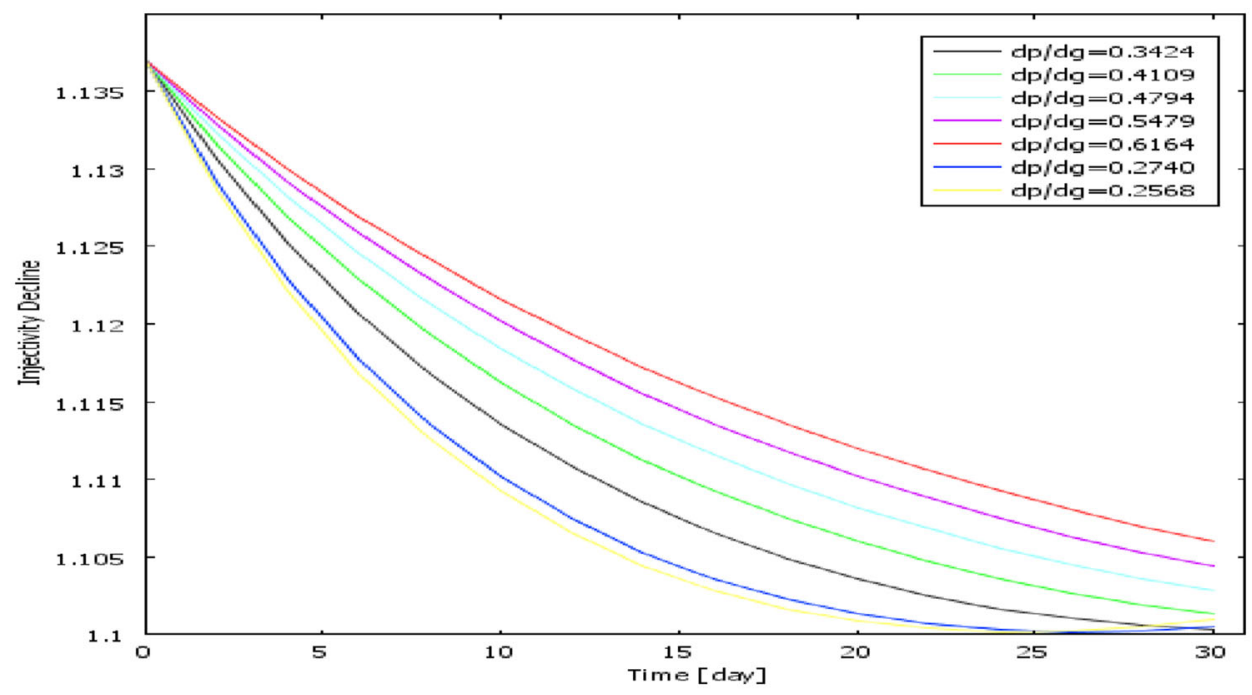

coefficients particle and grain size diameters and more importantly adsorption kinetics and produced water quality.

Acknowledgements The data were supplied by Department of Petroleum Resources (DPR) and Energy Technology Company in Houston, Texas, and this was well appreciated. Substantial data analysis was carried out by a simulation software supplied by Systems Engineering and Chemical and Petroleum Engineering faculty, and this was well appreciated as well. The authors express thanks to Division of Petroleum Regulator, Department of Petroleum Resources, and CNL/Energy Tech. Co. for access to data under their Local Technology Partnerships in PhD research work thesis and supported by the University of Lagos, Postgraduate School for granting publication of data for research and scholarly purpose.
Open Access This article is distributed under the terms of the Creative Commons Attribution 4.0 International License (http:// creativecommons.org/licenses/by/4.0/), which permits unrestricted use, distribution, and reproduction in any medium, provided you give appropriate credit to the original author(s) and the source, provide a link to the Creative Commons license, and indicate if changes were made.

\section{Appendix A: Well schematics for target wells}

See Figs. 25, 26, 27 and 28. 
Fig. 25 Well 217

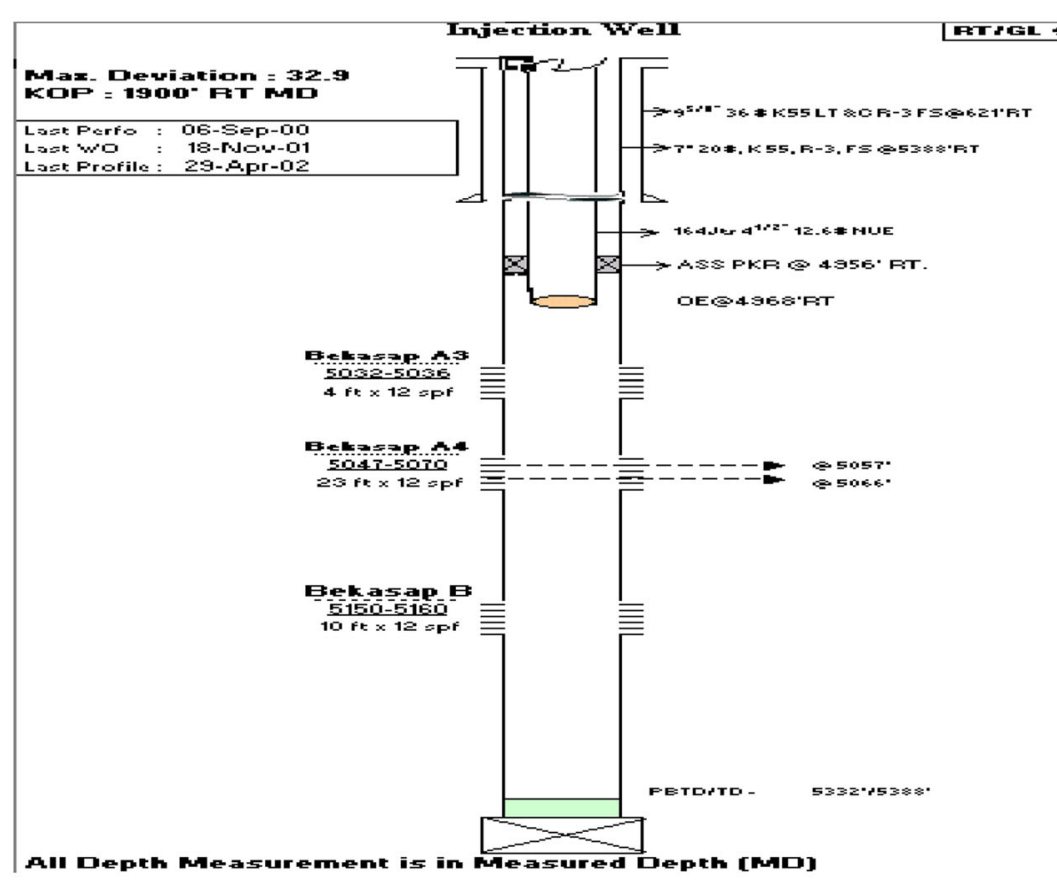

Fig. 26 Well 210

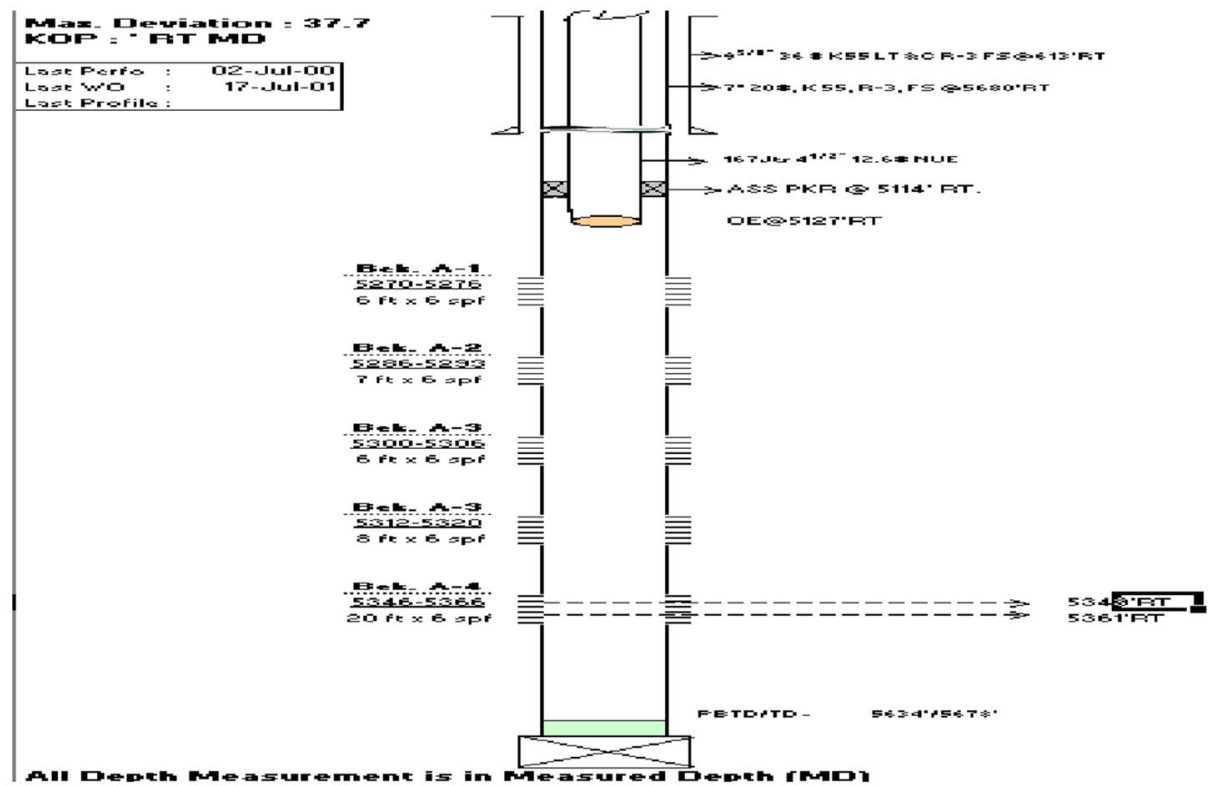


Fig. 27 Well 38

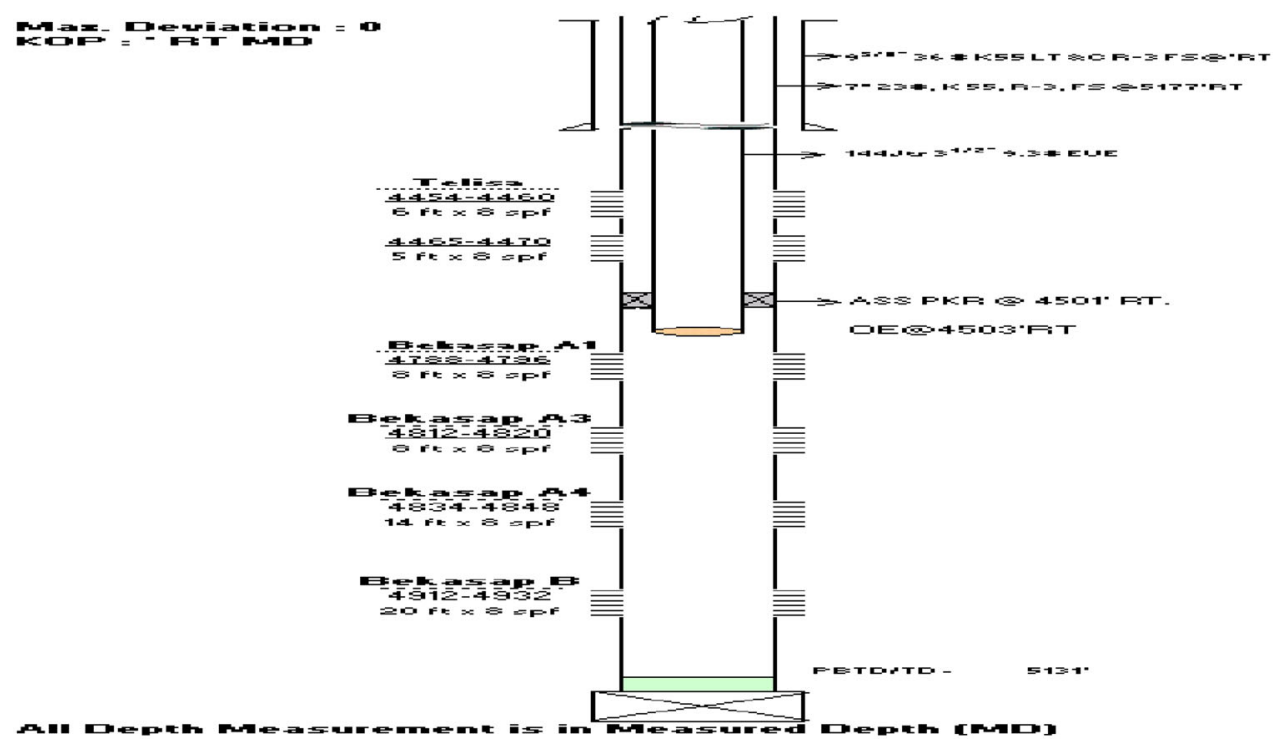

Fig. 28 Well 238

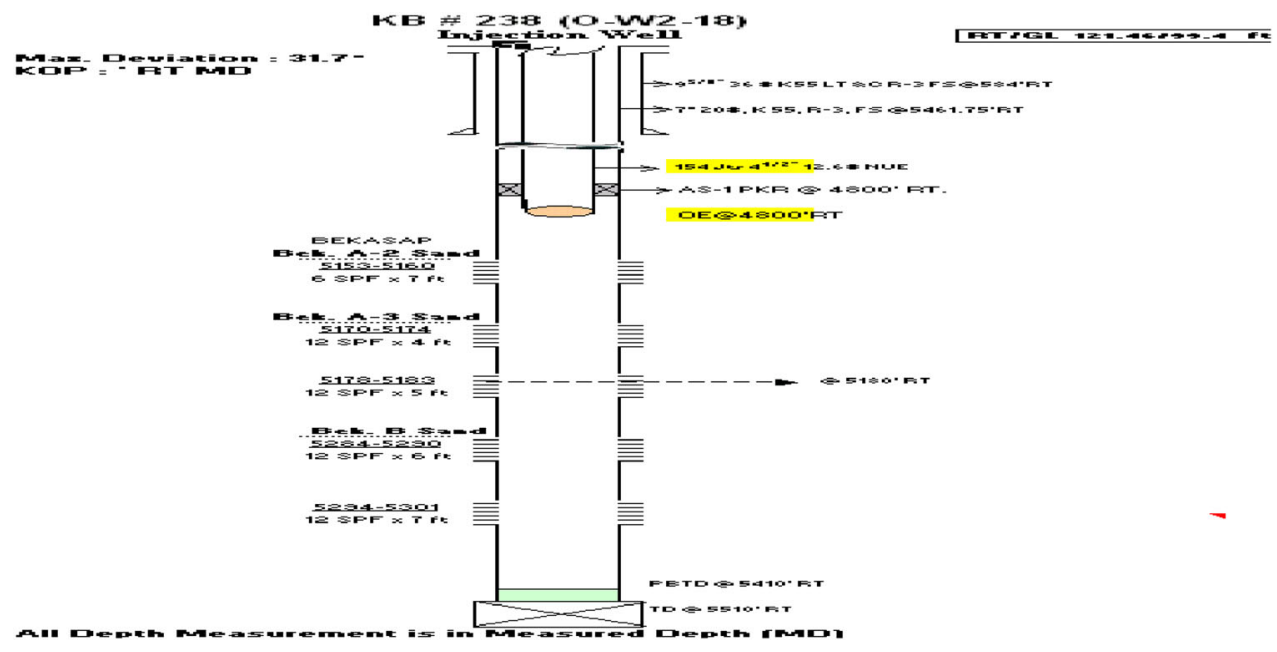

\section{References}

Abou-Sayed AS, Zaki KS, Wang GG, Sarfare MD (2005).A mechanistic model for formation damage and fracture propagation during water injection. In: Paper 94606 presented at the SPE European formation damage conference, Sheveningen

Abou-Sayed AS, Zaki KS, Wang GG, Sarfare MD, Harris MH (2007) Produced water management strategy and water injection best practices: design, performance and monitoring. SPEPO 22(1):59-68

Ajay S, Sharman MM (2007) A model for water injection into fracpacked wells. In: Paper SPE 110084 presented at the 2007 annual technical conference, Anaheim

Al-Abduwani FAH (2005) Internal filtration and external filter cake build-up in rock fracture stones. Ph.D. Dissertation, Delft University of Technology, Delft. ISBN 90-9020245-5

Al-Abduwani FAH, van den Broek WMGT, Currie PK (2001) Visual observation of produced water re-injection under laboratory conditions. In: Paper 68977 presented at the SPE European formation damage conference, The Hague
Altoef JE, Bedrikovetsky P, Gomes ACA, Siqueira AG, Desouza AI (2004) Effects of oil water mobility on injectivity impairment due to suspended solids. In: SPE Asia Pacific oil and gas conference

Barkman JH, Davidson DH (1972) Measuring water quality and predicting well impairment. J Pet Technol 24(7):865-873

Bedrikovetsky P, Furtado CA, de Souza ALS, Siquera FD (2007) Internal erosion in rocks during produced and sea water injection. In: SPE Paper 107513 presented at the SPE Europec/ EAGE annual conference and exhibition held in London, UK

Belfort G, Davis RH, Zydney AL (1994) The behavior of Suspensions and macromolecular solutions in cross flow filtration. Chem Eng Sci 45:3204-3209

Chang CK (1985) Water quality considerations in Malaysia's first water flood. J Pet Technol 37(9):1689-1698. doi:10.2118/12387-PA

Clifford PJ, Mellor DW, Jones TJ (1991) Water quality requirements for fractured injection wells. In: SPE 21439, presented at the SPE Middle East Oil Show, Nov, pp 851-862

Davidson DH (1979) Invasion and impairment of formation by particulates. In: Paper 8210, presented at the SPE annual technical conference and exhibition, Las Vegas 
De Zwart AH (2007) Investigation of clogging processes in unconsolidated aquifers near water supply wells. Ph.D. Dissertation, Delft University of Technology, Delft

Dong CY, Wu L, Wang AP (2010) Experimental simulation of gravel-packing in horizontal and highly deviated well. J China Univ Pet 34(2):74-82

Donaldson EC, Baker BA, Carroll HB (1977) Particle transport in sandstones. In: Paper SPE 6905, presented at the 1977 SPE, Annual Technical Conference and Exhibition, Denver, Colorado

Doresa R, Hussaina A, Katebaha M, Adhama S (2012) Advanced water treatment technologies for produced water. In: Proceedings of the 3rd international gas processing symposium

Farajzadeh R (2002) Produced water re-injection (PWRI)—an experimental investigation into internal filtration and external cake buildup. MSc Thesis, Delft University of Technology, Delft

Faruk C (2010) Non-isothermal permeability impairment by fines migration and deposition in porous media including dispersive transport. Transp Porous Media 85:233-258

Folarin F, An D, Caffrey S, Soh J, Sensen CW, Voordouw J, Jack T, Voordouw G (2013) Contribution of make-up water to the microbial community in an oilfield from which oil is produced by produced water re-injection. Int Biodeterior Biodegrad 81:44-50

Furtado CJA, Siquera AG, Souza ALS, Correa ACF, Mendes RA (2005) Produced water reinjection in petrobas fields: challenges and perspectives. In: Paper 94705 presented at the SPE Latin American and Caribbean petroleum engineering conference, Rio de Janeiro, Brazil

Gong B, Liang H, Xin Z, Li K (2013) Numerical studies on power generation from co-produced geothermal resources in oil fields and change in reservoir temperature. Renew Energy 50:722-731

Greenhill K (2002) Brazil frade fluid properties technical service report. EPTC TS02000099. 15 Nov

Guedes RG, Al-Abdouwani FA, Bedrikovetsky PG, Currie PK (2006) Injectivity decline under multiple particle capture mechanisms. In: SPE international symposium and exhibition on formation damage control. 15-17 February, Society of Petroleum Engineers, Lafayette, Louisiana, USA. doi:10.2118/98623-MS

Guo Q (2000) Update on the audit of available fracture models for PWRI. In: Presented to the participants meeting for the Produced Water Re-Injection JIP at Littleton, CO, 25-26 Apr 2000

Hustedt B, Zwarts D, Bjoerndal HP, Mastry R, van den Hoek PJ (2006) Induced fracturing in reservoir simulation: application of a new coupled simulator to water flooding field examples. In: Paper 102467 presented at SPE annual technical conference and exhibition held in San Antonio, Texas, USA

Idialu PO (2014) Modeling of adsorption kinetics, hydrodynamic dispersion and geochemical reaction of produced water reinjection (PWRI) in hydrocarbon aquifer. Ph.D. thesis, Department of System Engineering, University of Lagos

Iwasaki T (1937) Some notes on rock fracture filtration. J Am Water Works Assoc 29:1591

Khatib Z (2007) Produced water management: is it a future legacy or a business opportunity for field development. In: International petroleum technology conference (IPTC 11624), held in Dubai, UAE

Khodaverdian M, Sorop T, Postif S, van den Hoek PJ (2009) Polymer flooding in unconsolidated rock fracture formations: fracturing and geochemical considerations. In: Paper 121840 presented at the SPE Europe/EAGE annual conference and exhibition held in Amsterdam, The Netherlands

Lawal AK, Vesovic V (2010) Modeling asphaltene-induced formation damage in closed systems. In: SPE Paper 138497 presented in international petroleum exhibition and conference, Abu Dhabi

Lawal KA, Vesovic V, Edo SB (2011) Modeling permeability impairment in porous media due to asphaltene deposition under dynamic conditions. Energy Fuels 25:5647-5659
Li M, Li Y, Wang L (2011) Productivity prediction for gravel-packed horizontal well considering variable mass flow in wellbore. J China Univ Pet 35(3):89-92

Li Y, Li M, Qin G, Wu J, Wang W (2012) Numerical simulation study on gravel-packing layer damage by integration of innovative experimental observations. In: Paper 157927 presented in SPE heavy oil conference held in Calgary, Alberta, Canada

McDowell-Boyer LM, Hunt JR, Sitar N (1986) Particle transport through porous media. Water Resour Res 22:1901-1921

Meyer BR et al (2003a) Meyer fracturing simulators users guide appendices, 3rd edn. Meyer \& Associates Inc., Sulphur

Meyer BR et al (2003b) Meyer fracturing simulators users guide, 3rd edn. Meyer \& Associates Inc., Sulphur

Morita N, Whitfill DL, Massie I, Knudsen TW (1987a) Realistic study of sand production prediction -numerical approach. SPE 16989. In: Proceedings of the 1987 Annual Technical Conference and Exhibition. Dallas, September 27-30

Morita N, Whitfill DL, Fedde OP, Lvik TH (1987b) Realistic Sand Production Prediction: Analytical Approach. Paper SPE 16990, 62nd Annual Technical Conference and Exhibition of the Society of Petroleum Engineers Proc., Dallas, Texas

Obe I, Fashanu TA, Idialu PO, Akintola TO, Abhulimen KE (2017) Produced water re-injection in a non-fresh water aquifer with geochemical reaction, hydrodynamic molecular dispersion and adsorption kinetics controlling: model development and numerical simulation. Appl Water Sci 7(3):1169-1189. doi:10.1007/ s13201-016-0490-4

Ojukwu KI, van den Hoek PJ (2004) A new way to diagnose injectivity decline during fractured water injection by modifying conventional hall analysis. In: Paper 89376 presented at the SPE/ DOE Fourteenth symposium on improved oil recovery held in Tulsa, Oklahoma, USA

Pang S, Sharman MM (1994) A model for predicting injectivity decline in water injection wells. In: Paper SPE 28489 presented at the 1994 annual technical conference, New Orleans

Pang S, Sharma MM (1997) A model for predicting injectivity decline in water injection wells. SPE Form Eval 12(3):194-201. doi:10. 2118/28489-PA

Prasad KS, Bryant SL, Sharma MM (1999) Role of fracture face and formation plugging in injection well fracturing and injectivity decline. In: Paper 52731 presented at the 1999 SPE/EPA conference held in Austin, Texas

Sahni A, Kovacevich ST (2007) Produced water management alternatives for offshore environmental stewardship. In: Paper 108893 presented at the SPE Asia Pacific health, safety, security and environment conference and exhibition held in Bangkok, Thailand

Salehi MR, Settari A (2008) Velocity-based formation damage characterization method for produced water re-injection: application on masila block core flood tests. University of Calgary, Alberta

Shuler PJ, Subcaskey WJ (1997) Literature review: oily produced water reinjection. CPTC TM97000078 in March

Souza ALS, Figueiredo MW, Kuchpil C, Bezerra MC, Siquera AG Furtado C (2005) Water management in petrobras: developments and challenges. In: Paper OTC 17258 presented at the Offshore technology conference, Houston, Texas, USA

Todd A(1979) Review of permeability damage studies and related north sea water injection. In: Presented at the Society of petroleum engineers international symposium on oilfield and geothermal chemistry, Dallas

Van den Hoek PJ, Matsuura T, Dekroon M, Gheissary G (1996) Simulation of produced water re-injection under fracturing conditions. In: Paper 36846 presented at the SPE European petroleum conference held in Milan, Italy

Wang YF, Le TT (2008) Experimental study on the relationship between rock fracture particle migration and permeability in 
unconsolidated rock fracturestone reservoirs. J Oil Gas Technol 30(3):111-114

Wang LY, Gaa CX, Mbadinga SM, Zhou L, Liu JF, Gu JD, Mua BZ (2011) Characterization of an alkane-degrading methanogenic enrichment culture from production water of an oil reservoir after 274 days of incubation. Int Biodeterior Biodegrad 65:444-450

Sharma MM, Pang S, Wennberg KE, Morgenthaler LN (2000) Injectivity decline in water-injection wells: an offshore gulf of mexico case study. In: SPE Production \& Facilities, Feb, pp 6-13

Wehunt CD (2002) In situ stress and wellbore stability in the frade field, Brazil. Frade CPDEP Phase 2 report DR-AP-RP-021209 by GeoMechanics International, Inc.

Wennberg KE, Sharma MM (1997) Determination of filtration coefficient and transition time for water injection wells. In:
Paper 38181 presented at SPE European formation damage conference, The Hague

Yerramilli RC, Zitha PLJ, Yerramilli SS, Bedrikovetsky P (2013) A novel water injectivity model and experimental validation using ct scanned core-floods. In: Paper 165194, presented at the SPE European formation damage conference and exhibition held in Noorwijk, The Netherlands

Zeinijahromi A, Lemon P, Bedrikovetsky P (2011) Effects of induced fines migration on water cut during water flooding. J Pet Sci Eng 78:609-617

Zhang NS, Somerville JM, Todd AC (1993) An experimental investigation of the formation damage caused by produced oily water injection. In: Paper 26702 presented at the Offshore Europe, Aberdeen, United Kingdom 